Pure and Applied Mathematics Quarterly

Volume 8, Number 2

(Special Issue: In honor of

F. Thomas Farrell and Lowell E. Jones, Part 2 of 2)

$451-477,2012$

\title{
Split Injectivity of the Baum-Connes Assembly Map
}

\author{
David Rosenthal
}

\begin{abstract}
In this work, the continuously controlled techniques developed by Carlsson and Pedersen are used to prove that the Baum-Connes map is a split injection for groups satisfying certain geometric conditions.

Keywords: Baum-Connes map, $K$-theory, $C^{*}$-algebras, controlled topology.
\end{abstract}

\section{INTRODUCTION}

In [Hig00], Higson used analytic techniques to prove that the Baum-Connes map

$$
K K_{i}^{\Gamma}\left(C_{0}(\underline{\mathrm{E}} \Gamma) ; \mathbb{C}\right) \rightarrow K_{i}\left(C_{r}^{*} \Gamma\right),
$$

where $\Gamma$ is a discrete group and $\underline{\mathrm{E}} \Gamma$ denotes the universal space for $\Gamma$-actions with finite isotropy, is injective when $\underline{E} \Gamma$ satisfies certain geometric conditions. The goal of this paper is to use continuously controlled algebra to prove a strengthening of this result, namely that under these conditions, the Baum-Connes map is a split injection. This tells us that the left-hand side of the map is a direct summand of the right-hand side. Thus, a piece of $K_{i}\left(C_{r}^{*} \Gamma\right)$ is understood since there are tools available for calculating homology groups. Specifically, the following theorem is proved in this paper.

Received September 5, 2007.

2000 Mathematics Subject Classification.Primary 19K99, Secondary 18 F99. 
Theorem (Theorem 4.1). Let $\Gamma$ be a discrete group, and let $\underline{\mathrm{E}} \Gamma$ be a finite $\Gamma-C W$

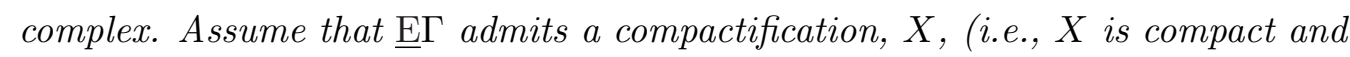
$\underline{\mathrm{E}} \Gamma$ is an open dense subset) such that

1. the $\Gamma$-action extends to $X$;

2. $X$ is metrizable;

3. $X^{G}$ is contractible for every finite subgroup $G$ of $\Gamma$;

4. $\mathrm{E}^{G}$ is dense in $X^{G}$ for every finite subgroup $G$ of $\Gamma$;

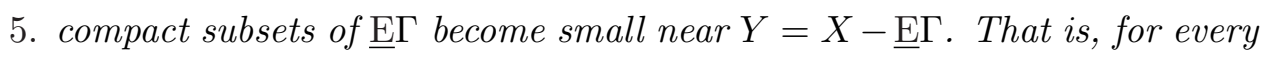
compact subset $K \subset \mathrm{E} \Gamma$ and for every neighborhood $U \subset X$ of $y \in Y$, there exists a neighborhood $V \subset X$ of $y$ such that $g \in \Gamma$ and $g K \cap V \neq \emptyset$ implies $g K \subset U$.

Then the Baum-Connes map, $K K_{i}^{\Gamma}\left(C_{0}(\underline{\mathrm{E}} \Gamma) ; \mathbb{C}\right) \rightarrow K_{i}\left(C_{r}^{*} \Gamma\right)$, is a split injection.

Boundaries of this type were first used by Farrell and Hsiang to prove the Novikov conjecture for fundamental groups of closed non-positively curved manifolds [FH81]. The proof of Theorem 4.1 uses continuously controlled algebra and is based on Carlsson and Pedersen's work in [CP95], where they split the assembly maps in algebraic $K$ - and $L$-theory for torsion free groups satisfying the same geometric conditions as are used here. Their results were generalized to groups with torsion in [Ros04, Ros06]. In these papers, the assembly map was split by realizing it as a map of fixed spectra. This allowed homotopy fixed sets to be used. More precisely, if $\mathbf{S} \rightarrow \mathbf{T}$ is a $\Gamma$-equivariant map of spectra, then the $\Gamma$-equivariant map $\underline{\mathrm{E}} \Gamma \rightarrow \bullet$ induces the diagram

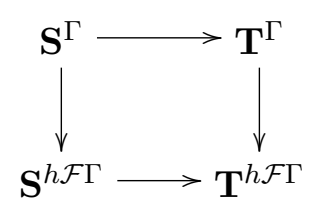

where $\mathbf{S}^{h \mathcal{F} \Gamma}$ denotes the set of all $\Gamma$-equivariant maps from $\underline{\mathrm{E}} \Gamma$ to $\mathbf{S}$. The homotopy fixed set, $\mathbf{S}^{h \mathcal{F} \Gamma}$, behaves well with respect to homotopy. In [CP95, Ros04, Ros06], the splittings were obtained by showing that the leftmost vertical map and the bottom map in the diagram were weak homotopy equivalences.

In order to enter the world of topological $K$-theory, additional structure must be added to the continuously controlled categories. In the process, the hope for realizing the Baum-Connes map as a map of fixed sets is lost. However, it is 
closely related to a map of fixed sets. Homotopy fixed sets can still be used to split the Baum-Connes map. The added structure also means that work must be done in order to extend the continuously controlled results from [CP95, Ros04].

Section 2 provides the background for the paper, including the basic definitions from continuously controlled algebra, a brief discussion of the $\mathbb{K}^{\text {Top }}$-functor, the continuously controlled formulation of the Baum-Connes map, and the necessary facts about homotopy fixed sets. In Section 3, the groundwork for the proof of the main theorem is laid. In this section, the continuously controlled algebra results from [CP95] that we require are extended to this setting. It turns out that in the proof of the main theorem it will be necessary to know that the reduced Steenrod homology (described in this section) of the quotient of a contractible compact metrizable space by a finite group is trivial. This is proved making use of the Conner Conjecture. In Section 4, the main theorem is proved. The most difficult step in the proof is handled by filtering the space $X$ by conjugacy classes of fixed sets.

\section{Preliminaries}

2.1. Continuously Controlled Algebra. Let $\Gamma$ be a discrete group, $X$ a $\Gamma$ space, and $Y$ a closed $\Gamma$-invariant subspace of $X$. Let $E=X-Y$. The complex vector space with basis $E \times \Gamma \times \mathbb{N}$ is denoted $\mathbb{C}[E \times \Gamma]^{\infty}$. If $H$ is a vector subspace of $\mathbb{C}[E \times \Gamma]^{\infty}$, denote $H \cap \mathbb{C}[\{x\} \times \Gamma]^{\infty}$ by $H_{x}$, where $x \in E$. The continuously controlled category $\mathcal{B}(X, Y ; \mathbb{C})$, has objects $H$, where

(i) $H=\bigoplus_{x \in E} H_{x}$;

(ii) $H_{x}$ is a finite dimensional complex vector space with basis contained in $\{x\} \times \Gamma \times \mathbb{N}$

(iii) $\left\{x \in E \mid H_{x} \neq 0\right\}$ is locally finite in $E$.

Morphisms are all linear operators $\phi: H \rightarrow K$, that are continuously controlled. This means that for every $y \in Y$ and every neighborhood $U \subseteq X$ of $y$, there exists a neighborhood $V \subseteq X$ of $y$ such that the components of $\phi, \phi_{z}^{x}: H_{x} \rightarrow K_{z}$ and $\phi_{x}^{z}: K_{z} \rightarrow H_{x}$, are zero whenever $x \in V$ and $z \notin U$.

The definition of this category can also be made for an arbitrary ring. Although such generality is not needed in this paper, we will encounter situations in which the complex group ring $\mathbb{C} G$ is used, where $G$ is a subgroup of $\Gamma$. The only 
changes in the definition are that the objects of $\mathcal{B}(X, Y ; \mathbb{C} G)$ are free $\mathbb{C} G$-modules, where each $H_{x}$ is a finite dimensional free $\mathbb{C} G$-module, and morphisms have the additional feature that their components are $\mathbb{C} G$-homomorphisms.

Note that $\mathbb{C}[E \times \Gamma]^{\infty}$ comes equipped with a free $\Gamma$-action. This action puts an interesting action of $\Gamma$ on $\mathcal{B}(X, Y ; \mathbb{C})$. If we assume that $\Gamma$ acts on $E$ with finite isotropy, then the fixed category $\mathcal{B}^{\Gamma}(X, Y ; \mathbb{C})$ has those objects $H$ in $\mathcal{B}(X, Y ; \mathbb{C})$ that satisfy the conditions

1. $H_{\gamma x} \cong H_{x}$ for every $\gamma \in \Gamma$, and

2. $H_{x}$ is a finitely generated free $\mathbb{C} \Gamma_{x}$-module.

This implies that $\bigoplus_{x^{\prime} \in[x]} H_{x^{\prime}}$ is a finitely generated free $\mathbb{C} \Gamma$-module, where $[x]=$ $\{\gamma x \mid \gamma \in \Gamma\}$. The morphisms in $\mathcal{B}^{\Gamma}(X, Y ; \mathbb{C})$ are those morphisms $\phi$ in $\mathcal{B}(X, Y ; \mathbb{C})$ that are $\Gamma$-equivariant, i.e., $\gamma \phi_{y}^{x} \gamma^{-1}=\phi_{\gamma y}^{\gamma x}$ for all $\gamma \in \Gamma$ and all $x, y \in E$. Note that if a finite group $H$ acts trivially on $E$ then the fixed category $\mathcal{B}^{H}(X, Y ; \mathbb{C})$ is equivalent to $\mathcal{B}(X, Y ; \mathbb{C} H)$.

In order to discuss topological $K$-theory, our categories need to be modified. The category $C_{r}^{*} \mathcal{B}(X, Y ; \mathbb{C})$ is defined to have the same objects as $\mathcal{B}(X, Y ; \mathbb{C})$, but morphism sets are defined as follows. Every object, $H$, has a unique Hilbert space completion, $\mathcal{H}$, and every bounded morphism, $T: H \rightarrow K$, extends uniquely to a bounded operator, $T: \mathcal{H} \rightarrow \mathcal{K}$. The set of morphisms from $H$ to $K$ in $C_{r}^{*} \mathcal{B}(X, Y ; \mathbb{C})$ is obtained by first considering the subgroup of continuously controlled morphisms from $H$ to $K$ that are bounded operators and then taking the closure of their extensions inside $\mathfrak{B}(\mathcal{H}, \mathcal{K})$.

Notice that in $C_{r}^{*} \mathcal{B}(X, Y ; \mathbb{C})$ we no longer have strict control over morphisms. However, any morphism in $C_{r}^{*} \mathcal{B}(X, Y ; \mathbb{C})$ can be approximated arbitrarily closely by a controlled morphism in $\mathcal{B}(X, Y ; \mathbb{C})$. Since group actions are continuous, we similarly define $C_{r}^{*} \mathcal{B}^{\Gamma}(X, Y ; \mathbb{C})$.

Remark 2.1. At first glance $C_{r}^{*} \mathcal{B}^{\Gamma}(X, Y ; \mathbb{C})$ appears to be the fixed category $\left(C_{r}^{*} \mathcal{B}(X, Y ; \mathbb{C})\right)^{\Gamma}$, but this is not the case in general. A morphism in the category $C_{r}^{*} \mathcal{B}^{\Gamma}(X, Y ; \mathbb{C})$ is a limit of $\Gamma$-equivariant continuously controlled morphisms, whereas in the fixed category $\left(C_{r}^{*} \mathcal{B}(X, Y ; \mathbb{C})\right)^{\Gamma}$ there can be $\Gamma$-equivariant morphisms that are limits of non-equivariant continuously controlled morphisms. There is, however, an inclusion functor from $C_{r}^{*} \mathcal{B}^{\Gamma}(X, Y ; \mathbb{C})$ to $\left(C_{r}^{*} \mathcal{B}(X, Y ; \mathbb{C})\right)^{\Gamma}$. If $\Gamma$ is a finite group, then this functor is an equivalence. 
Lemma 2.2. If $\Gamma$ is a finite group, then $C_{r}^{*} \mathcal{B}^{\Gamma}(X, Y ; \mathbb{C}) \cong\left(C_{r}^{*} \mathcal{B}(X, Y ; \mathbb{C})\right)^{\Gamma}$.

Proof. We need only resolve the issue mentioned above. Let $T$ be a morphism in $\left(C_{r}^{*} \mathcal{B}(X, Y ; \mathbb{C})\right)^{\Gamma}$. Then $T$ is a limit of continuously controlled bounded operators $\left\{T_{n}\right\}$. Let

$$
S_{n}=\frac{1}{|\Gamma|} \sum_{g \in \Gamma} g T_{n} g^{-1} .
$$

Then $S_{n}$ is a $\Gamma$-equivariant continuously controlled bounded operator, and since $T$ is $\Gamma$-equivariant, $\left\{S_{n}\right\}$ converges to $T$.

Two controlled categories that are of particular interest to us are $\mathcal{B}(C X, C Y \cup$ $\left.X, p_{X} ; \mathbb{C}\right)$ and $\mathcal{B}\left(\Sigma X, \Sigma Y, p_{X} ; \mathbb{C}\right)$, where $C X$ denotes the cone of $X, \Sigma X$ denotes the unreduced suspension of $X$, and $p_{X}: X \times(0,1) \rightarrow X$ is the projection map. These categories have the same objects as $\mathcal{B}(C X, C Y \cup X ; \mathbb{C})$ and $\mathcal{B}(\Sigma X, \Sigma Y ; \mathbb{C})$ respectively, but their control conditions on morphisms differ along $Y \times(0,1)$, where they are only required to be $p_{X}$-controlled. This means that for every $(y, t) \in Y \times(0,1)$ and every neighborhood $U \subseteq X$ of $y$, there is a neighborhood $V$ of $(y, t)$ such that $\phi_{b}^{a}=0$ and $\phi_{a}^{b}=0$ whenever $a \in V \cap p_{X}^{-1}(U)$ and $b \notin p_{X}^{-1}(U)$.

In [Kar70], Karoubi introduced the notion of an $\mathcal{A}$-filtered additive category, where $\mathcal{A}$ is a full subcategory of $\mathcal{U}$. The associated quotient category, $\mathcal{U} / \mathcal{A}$, has the same objects as $\mathcal{U}$, but two morphisms are identified if their difference factors through $\mathcal{A}$. The support at infinity of an object $H$ in $\mathcal{B}(X, Y ; \mathbb{C})$, denoted $\operatorname{supp}_{\infty}(H)$, is the set of limit points of $\left\{x \mid H_{x} \neq 0\right\}$. If $C$ is a closed subspace of $Y$, then the category $\mathcal{B}(X, Y ; \mathbb{C})_{C}$ is the full subcategory of $\mathcal{B}(X, Y ; \mathbb{C})$ on objects $H$ with $\operatorname{supp}_{\infty}(H) \subseteq C$. The category $\mathcal{B}(X, Y ; \mathbb{C})$ is $\mathcal{B}(X, Y ; \mathbb{C})_{C}$-filtered. If $W$ is an open subspace of $Y$, then the germ category $\mathcal{B}(X, Y ; \mathbb{C})^{W}$ has the same objects as $\mathcal{B}(X, Y ; \mathbb{C})$, but morphisms are identified if they agree in a neighborhood of $W$. It is equivalent to the quotient category $\mathcal{B}(X, Y ; \mathbb{C}) / \mathcal{B}(X, Y ; \mathbb{C})_{C}$, when $C=Y-W$ [CP95]. The category $C_{r}^{*} \mathcal{B}(X, Y ; \mathbb{C})_{C}$ is the full subcategory of $C_{r}^{*} \mathcal{B}(X, Y ; \mathbb{C})$ on objects $H$ with $\operatorname{supp}_{\infty}(H) \subseteq C$, and $C_{r}^{*} \mathcal{B}(X, Y ; \mathbb{C})$ is $C_{r}^{*} \mathcal{B}(X, Y ; \mathbb{C})_{C}$-filtered. Two morphisms in the corresponding quotient category are identified if their difference can be approximated arbitrarily closely by continuously controlled bounded operators that factor through objects in $\mathcal{B}(X, Y ; \mathbb{C})_{C}$. This is equivalent to saying that two morphisms are identified if their difference can be approximated arbitrarily closely by continuously controlled bounded operators that are zero in a neighborhood of $W=Y-C$. Thus, we will denote this 
quotient category by $C_{r}^{*} \mathcal{B}(X, Y ; \mathbb{C})^{Y-C}$. The reason for studying these Karoubi filtrations is that the sequence

$$
C_{r}^{*} \mathcal{B}(X, Y ; \mathbb{C})_{C} \rightarrow C_{r}^{*} \mathcal{B}(X, Y ; \mathbb{C}) \rightarrow C_{r}^{*} \mathcal{B}(X, Y ; \mathbb{C})^{Y-C}
$$

induces a fibration of spectra after applying $\mathbb{K}^{\text {Top }}$. Such sequences are the main tool in this theory. The above discussion also works for fixed categories if we use $\Gamma$-invariant subspaces.

When analyzing assembly maps the categories $\mathcal{B}(E \times(0,1], E \times 1 ; \mathbb{C})$ and $\mathcal{B}(E \times(0,1], E \times 1 ; \mathbb{C})^{E \times 1}$, and their $C_{r}^{*}$-counterparts, are used. For notational convenience, we denote these categories by $\mathcal{B}(E \times(0,1) ; \mathbb{C})$ and $\mathcal{B}(E \times(0,1) ; \mathbb{C})^{>0}$.

2.2. The $\mathbb{K}^{\text {Top }}$ Functor. There are two functors called $\mathbb{K}^{\text {Top }}$, one from the category of $C^{*}$-algebras to the category of spectra and the other from the category of $C^{*}$-categories to the category of spectra. The two functors are closely related. The definition of $\mathbb{K}^{\text {Top }}$ of a $C^{*}$-algebra is simple to state. For a given $C^{*}$-algebra $A, \mathbb{K}^{\operatorname{Top}}(A)=\Omega^{-1} \mathbb{U}(A)$, where $\mathbb{U}(A)$ is the $\Omega$-spectrum whose first term is the infinite loop space $U(A)=\{u \in A \otimes \mathfrak{K} \mid 1+u$ is a unitary in the unitization of $A \otimes \mathfrak{K}\}$, which satisfies Bott periodicity [HPR97]. The definition of $\mathbb{K}^{\text {Top }}$ of a $C^{*}$-category is not as easy to state and will not be presented here (an excellent reference is [HP04]). However, the catch phrase is that the $K$-theory of a $C^{*}$-category is the direct limit of the $K$-theory of the endomorphisms of the objects of the category. For example, $\mathbb{K}^{\operatorname{Top}}\left(C_{r}^{*} \mathcal{B}(\bullet, \emptyset ; \mathbb{C} \Gamma)\right) \simeq \mathbb{K}^{\operatorname{Top}}\left(C_{r}^{*} \Gamma\right)$.

In [HPR97] the $K$-theory of continuously controlled $C^{*}$-categories was identified with a particular $C^{*}$-algebra constructed by John Roe. This correspondence is used to establish certain properties of the $\mathbb{K}^{\text {Top }}$-functor that are necessary for using the continuously controlled techniques. Under this identification, a filtered subcategory corresponds to a closed two-sided ideal in the Roe algebra. Since a short exact sequence of $C^{*}$-algebras induces a fibration of spectra after applying $\mathbb{K}^{\text {Top }}[$ HPR97, 4.4], the Karoubi filtration

$$
C_{r}^{*} \mathcal{B}(X, Y ; \mathbb{C})_{C} \rightarrow C_{r}^{*} \mathcal{B}(X, Y ; \mathbb{C}) \rightarrow C_{r}^{*} \mathcal{B}(X, Y ; \mathbb{C})^{Y-C}
$$

also yields a fibration of spectra (up to homotopy) after applying $\mathbb{K}^{\text {Top }}$.

It follows from the definition that $\mathbb{K}^{\text {Top }}$ of a countable $C^{*}$-direct sum of $C^{*}$ algebras $\bigoplus_{i} A_{i}$ is equivalent to the countable direct product $\prod_{i} \mathbb{K}^{\mathrm{Top}}\left(A_{i}\right)$. This 
implies that $\mathbb{K}^{\text {Top }}$ of a countable $C^{*}$-direct sum of $C^{*}$-categories $\bigoplus_{i} \mathcal{A}_{i}$ is equivalent to the countable direct product $\prod_{i} \mathbb{K}^{\mathrm{Top}}\left(\mathcal{A}_{i}\right)$. (The $C^{*}$-category $\bigoplus_{i} \mathcal{A}_{i}$ has the same objects as the product category $\prod_{i} \mathcal{A}_{i}$, but a morphism $T=\left\{T_{i}\right\}$ in $\bigoplus_{i} \mathcal{A}_{i}$ is a uniformly bounded sequence of morphisms.) The final property that we require is that $\mathbb{K}^{\text {Top }}$ applied to a fixed $C^{*}$-category $\mathcal{A}^{\Gamma}$ is equivalent to $\mathbb{K}^{\operatorname{Top}}(\mathcal{A})^{\Gamma}$

2.3. The Baum-Connes Assembly Map. Hambleton and Pedersen showed that the continuously controlled assembly map in topological $K$-theory

$$
\Omega \mathbb{K}^{\mathrm{Top}}\left(C_{r}^{*} \mathcal{B}^{\Gamma}(\underline{\mathrm{E}} \Gamma \times(0,1) ; \mathbb{C})^{>0}\right) \rightarrow \Omega \mathbb{K}^{\mathrm{Top}}\left(C_{r}^{*} \mathcal{B}(\bullet \times(0,1) ; \mathbb{C} \Gamma)^{>0}\right),
$$

induced by collapsing the universal space for $\Gamma$ actions with finite isotropy, $\underline{\mathrm{E}} \Gamma$, to a point, is equivalent (at the level of homotopy) to the Baum-Connes map, $K K_{i}^{\Gamma}\left(C_{0}(\underline{\mathrm{E}} \Gamma) ; \mathbb{C}\right) \rightarrow K_{i}\left(C_{r}^{*} \Gamma\right)[\mathrm{HP} 04$, Theorem 7.6]. When $\underline{\mathrm{E}} \Gamma$ admits a compactification $X$ that satisfies the conditions of our main theorem, the Baum-Connes assembly map can also be formulated using the functor

$$
C_{r}^{*} \mathcal{B}^{\Gamma}\left(C X, C Y \cup X, p_{X} ; \mathbb{C}\right) \rightarrow C_{r}^{*} \mathcal{B}^{\Gamma}\left(\Sigma X, \Sigma Y, p_{X} ; \mathbb{C}\right),
$$

produced by collapsing $X$ to a point (see Remark 3.7). If this was a map between fixed categories, then we would be able to use generalized fixed sets to split it as in [CP95, Ros04]. However, as we saw in Remark 2.1, these categories are not necessarily fixed categories. Nonetheless, it will still be possible to use the techniques that were developed for splitting assembly maps that are induced by maps of fixed sets.

2.4. Generalized Fixed Sets. In this section, we recall some facts about generalized homotopy fixed sets in the category of spectra. Let $\mathbf{S}$ be a spectrum with $\Gamma$-action. The fixed set $\mathbf{S}^{\Gamma}$, can be identified with the $\operatorname{set} \operatorname{Map}_{\Gamma}(\bullet, \mathbf{S})$ of $\Gamma$-equivariant maps from a point into $\mathbf{S}$. Let $\mathcal{F}$ be a family of subgroups of $\Gamma$ that is closed under conjugation and under the operation of taking subgroups. Let $\mathrm{E}_{\mathcal{F}} \Gamma$ be the universal space for $\Gamma$ actions with isotropy in $\mathcal{F}$. This space is a $\Gamma$-CW complex that is characterized by the fact that $\left(\mathrm{E}_{\mathcal{F}} \Gamma\right)^{G}$ is contractible for every $G$ in $\mathcal{F}$ and is empty otherwise. The $\mathcal{F}$-homotopy fixed point spectrum of $\mathbf{S}$ is defined to be

$$
\mathbf{S}^{h \mathcal{F} \Gamma}=\operatorname{Map}_{\Gamma}\left(\mathrm{E}_{\mathcal{F}} \Gamma, \mathbf{R}(\mathbf{S})\right)
$$


where $\mathbf{R}$ is a fibrant replacement functor from the category of spectra to the category of $\Omega$-spectra. For a construction of this functor see, for example, [LRV03, Section 2]. The fibrant replacement functor comes equipped with a natural weak equivalence $\mathbf{S} \rightarrow \mathbf{R}(\mathbf{S})$, and if $\mathbf{S}$ is a spectrum with $\Gamma$-action then so is $\mathbf{R}(\mathbf{S})$. Furthermore, $\mathbf{R}$ commutes with taking fixed points, i.e., $(\mathbf{R}(\mathbf{S}))^{\Gamma}=\mathbf{R}\left(\mathbf{S}^{\Gamma}\right)$.

The $\Gamma$-equivariant map $\mathrm{E}_{\mathcal{F}} \Gamma \rightarrow \bullet$ induces the following commutative diagram:

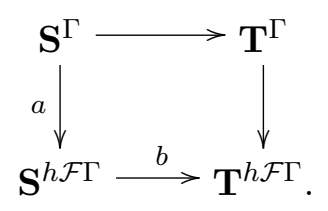

If $a$ and $b$ are shown to be weak homotopy equivalences, then at the level of homotopy, the map $\mathbf{S}^{\Gamma} \rightarrow \mathbf{T}^{\Gamma}$ will have successfully been split. In [CP95, Ros04, Ros06], such a diagram was used to split the assembly maps in algebraic $K$ - and $L$-theory by realizing them as maps of fixed sets. Despite the fact that we have not realized the Baum-Connes assembly map as a map of fixed sets, the above diagram can still be used (see Section 4.1). The next two lemmas play a key role in such an approach. Their proofs can be found in [BR07, Lemma 7.1].

Lemma 2.3. Let $F: \mathbf{S} \rightarrow \mathbf{T}$ be an equivariant map of spectra with $\Gamma$-action. Assume that $F^{G}: \mathbf{S}^{G} \rightarrow \mathbf{T}^{G}$ is a weak homotopy equivalence for every $G$ in $\mathcal{F}$. Then $\mathbf{S}^{h \mathcal{F} \Gamma} \rightarrow \mathbf{T}^{h \mathcal{F} \Gamma}$ is a weak homotopy equivalence.

Lemma 2.4. Let $\mathbf{B}$ be an $\Omega$-spectrum with $G$-action, where $G$ is in $\mathcal{F}$, and let $\Gamma$ act on $\mathbf{S}=\prod_{\Gamma / G} \mathbf{B}$ by identifying $\mathbf{S}$ with $\operatorname{Map}_{G}(\Gamma, \mathbf{B}) \quad$ (in which $(\gamma f)(x)=$ $f\left(\gamma^{-1} x\right)$, where $\left.\gamma \in \Gamma\right)$. Then $\mathbf{S}^{\Gamma} \rightarrow \mathbf{S}^{h \mathcal{F} \Gamma}$ is a weak homotopy equivalence.

\section{KEY FACTS}

3.1. $C^{*}$-algebras and Controlled Categories. In order to handle topological $K$-theory, additional structure needed to be placed on the continuously controlled categories. Recall that morphisms in $C_{r}^{*} \mathcal{B}^{\Gamma}(X, Y ; \mathbb{C})$ are required to be bounded operators. Furthermore, morphism sets are completed, so strict control is lost. Therefore we must verify that the various results that we need from [CP95, Ros04] can be extended to their $C_{r}^{*}$-counterparts. In general this is not so easy. For example, a functor between two controlled categories will extend to a functor in the $C_{r}^{*}$-setting if it sends bounded operators to bounded operators and Cauchy 
sequences of bounded operators to Cauchy sequences. However, checking whether or not a given functor possess these properties is non-trivial. Luckily, the various functors used in [CP95, Ros04] are defined in such a way that they are guaranteed to extend to their $C_{r}^{*}$-analogues.

A function $f:(X, Y) \rightarrow\left(X^{\prime}, Y^{\prime}\right)$ is eventually continuous if for every compact $K \subset X^{\prime}-Y^{\prime}, f^{-1}(K)$ has compact closure in $X-Y, f(X-Y) \subset X^{\prime}-Y^{\prime}$, and $f$ is continuous on $Y$. In [CP95, Proposition 1.16], it is proved that an eventually continuous function $f:(X, Y) \rightarrow\left(X^{\prime}, Y^{\prime}\right)$, induces a functor $U_{f}$ : $\mathcal{B}(X, Y ; \mathbb{C}) \rightarrow \mathcal{B}\left(X^{\prime}, Y^{\prime} ; \mathbb{C}\right)$ (well-defined up to natural equivalence), defined as follows. An object $H$ is sent to $U_{f}(H)$, where $\left(U_{f}(H)\right)_{x^{\prime}}=\bigoplus_{x \in f^{-1}\left(x^{\prime}\right)} H_{x}$, and comes equipped with a unitary operator $U_{H}: H \rightarrow U_{f}(H)$, defined by $U_{H}(v)_{x^{\prime}}=$ $\left(v_{x}\right)_{x \in f^{-1}\left(x^{\prime}\right)}$, where $v \in H$. If $T: H \rightarrow K$ is a continuously controlled morphism, then $U_{f}(T)=U_{K} T U_{H}^{-1}$. The unitary $U_{H}$ extends uniquely to a unitary, also denoted by $U_{H}$, on the Hilbert space completions of $H$ and $U_{f}(H)$. Now it is easy to see that $U_{f}$ extends to a functor $\mathcal{U}_{f}: C_{r}^{*} \mathcal{B}(X, Y ; \mathbb{C}) \rightarrow C_{r}^{*} \mathcal{B}\left(X^{\prime}, Y^{\prime} ; \mathbb{C}\right)$, where $\mathfrak{U}_{f}(H)=U_{f}(H)$, and $\mathfrak{U}_{f}(T)=U_{K} T U_{H}^{-1}$ when $T$ is a morphism from $H$ to $K$ in $C_{r}^{*} \mathcal{B}(X, Y ; \mathbb{C})$.

The remainder of this section is devoted to proving the $C_{r}^{*}$-versions of the results in [CP95] that we will need, making frequent use of unitary functors like the ones induced by eventually continuous maps. Throughout this section assume that $X$ is a compact metrizable space, $Y$ is a closed nowhere dense subset, and let $E=X-Y$.

Lemma 3.1. If $f_{1}, f_{2}:(X, Y) \rightarrow\left(X^{\prime}, Y^{\prime}\right)$ are eventually continuous maps and $\left.f_{1}\right|_{Y}=\left.f_{2}\right|_{Y}$, then $\mathcal{U}_{f_{1}}$ and $\mathcal{U}_{f_{2}}$ are naturally equivalent functors.

Proof. Let $H$ be an object in $C_{r}^{*} \mathcal{B}(X, Y ; \mathbb{C})$. Let $U_{H}: H \rightarrow \mathcal{U}_{f_{1}}(H)$ be the unitary operator associated to $H$ by $\mathcal{U}_{f_{1}}$, and let $V_{H}: H \rightarrow \mathcal{U}_{f_{2}}(H)$ be the unitary operator associated to $H$ by $\mathcal{U}_{f_{2}}$. Then the natural equivalence, $\eta: \mathcal{U}_{f_{1}} \rightarrow \mathcal{U}_{f_{2}}$, is given by $\eta(H)=V_{H} \circ U_{H}^{-1}$.

Proposition 3.2. There is a natural equivalence

$$
\mathcal{U}_{f}: C_{r}^{*} \mathcal{B}(X, Y ; \mathbb{C}) \rightarrow C_{r}^{*} \mathcal{B}(C Y, Y ; \mathbb{C})
$$


That is, if $X^{\prime}$ is a compact metrizable space, $Y^{\prime}$ is a closed nowhere dense subset, and $h:(X, Y) \rightarrow\left(X^{\prime}, Y^{\prime}\right)$ is an eventually continuous map, then

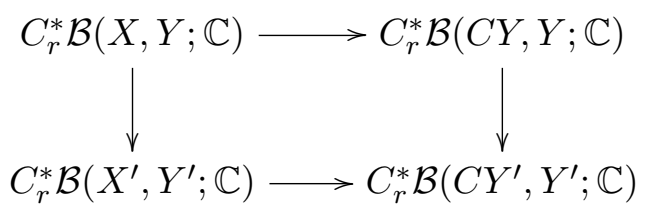

commutes up to natural equivalence.

Proof. There exist eventually continuous maps $f:(X, Y) \rightarrow(C Y, Y)$ and $g:$ $(C Y, Y) \rightarrow(X, Y)$ such that $\left.f\right|_{Y}=\left.g\right|_{Y}=1_{Y}$ [CP95, Theorem 1.23]. By Lemma $3.1, \mathcal{U}_{f}$ is an equivalence of categories with inverse $\mathcal{U}_{g}$.

Let $h:(X, Y) \rightarrow\left(X^{\prime}, Y^{\prime}\right)$ be an eventually continuous map. Define $f^{\prime}$ : $\left(X^{\prime}, Y^{\prime}\right) \rightarrow\left(C Y^{\prime}, Y^{\prime}\right)$ as in [CP95, Theorem 1.23]. Then $\left.(C h \circ f)\right|_{Y}=\left.\left(f^{\prime} \circ h\right)\right|_{Y}$. So by Lemma 3.1, $\mathcal{U}_{C h} \circ \mathcal{U}_{f}$ and $\mathcal{U}_{f^{\prime}} \circ \mathcal{U}_{h}$ are naturally equivalent.

Lemma 3.3. $C_{r}^{*} \mathcal{B}(X, Y ; \mathbb{C})^{W}=C_{r}^{*} \mathcal{B}(X-(Y-W), W ; \mathbb{C})^{W}$.

Proof. The two categories have precisely the same objects. Consider the functor from $C_{r}^{*} \mathcal{B}(X, Y ; \mathbb{C})^{W}$ to $C_{r}^{*} \mathcal{B}(X-(Y-W), W ; \mathbb{C})^{W}$ induced by forgetting control along $Y-W$. To show that the functor is one-to-one on morphism sets, consider a morphism $T$ in $C_{r}^{*} \mathcal{B}(X, Y ; \mathbb{C})$ whose image in $C_{r}^{*} \mathcal{B}(X-(Y-W), W ; \mathbb{C})^{W}$ is zero. This means that $T$ can be approximated arbitrarily closely by continuously controlled bounded operators that factor through objects that are zero on a neighborhood of $W$. Therefore, $T$ is identified with the zero morphism in $C_{r}^{*} \mathcal{B}(X, Y ; \mathbb{C})^{W}$.

To show that the functor is onto on morphism sets, let $T$ represent a morphism in $C_{r}^{*} \mathcal{B}(X-(Y-W), W ; \mathbb{C})^{W}$. By [CP95, Proof of Lemma 1.32], any morphism in $C_{r}^{*} \mathcal{B}(X-(Y-W), W ; \mathbb{C})^{W}$ that can be represented by a continuously controlled operator is in the image of the functor. Since $T$ can be approximated arbitrarily well by continuously controlled operators, $T$ is related to a limit of morphisms in the image of the functor.

Lemma 3.4. $C_{r}^{*} \mathcal{B}\left(C X, C Y \cup X, p_{X} ; \mathbb{C}\right)^{E}=C_{r}^{*} \mathcal{B}(E \times(0,1) ; \mathbb{C})^{>0}$.

Proof. By [CP95, Proof of Lemma 2.4], the forgetful functor from $\mathcal{B}(C X, C Y \cup$ $X ; \mathbb{C})^{E}$ to $\mathcal{B}\left(C X, C Y \cup X, p_{X} ; \mathbb{C}\right)^{E}$ is an equivalence of categories. As in the proof 
of Lemma 3.3, this equivalence extends to one between the $C_{r}^{*} \mathcal{B}(C X, C Y \cup X ; \mathbb{C})^{E}$ and $C_{r}^{*} \mathcal{B}\left(C X, C Y \cup X, p_{X} ; \mathbb{C}\right)^{E}$. Finally, $C_{r}^{*} \mathcal{B}(C X, C Y \cup X ; \mathbb{C})^{E}=C_{r}^{*} \mathcal{B}(E \times$ $(0,1) ; \mathbb{C})^{>0}$ by Lemma 3.3 .

Lemma 3.5. Let $\Gamma$ be a group acting on $X$ such that $Y$ is $\Gamma$-invariant. Then

$$
\mathbb{K}^{\mathrm{Top}}\left(C_{r}^{*} \mathcal{B}^{\Gamma}\left(C X, C Y \cup X, p_{X} ; \mathbb{C}\right)\right) \simeq \mathbb{K}^{\mathrm{Top}}\left(C_{r}^{*} \mathcal{B}^{\Gamma}\left(C X, C Y \cup X, p_{X} ; \mathbb{C}\right)^{E}\right) .
$$

Proof. The Karoubi filtration $C_{r}^{*} \mathcal{B}^{\Gamma}\left(C X, C Y \cup X, p_{X} ; \mathbb{C}\right)_{C Y} \rightarrow C_{r}^{*} \mathcal{B}^{\Gamma}(C X, C Y \cup$ $\left.X, p_{X} ; \mathbb{C}\right) \rightarrow C_{r}^{*} \mathcal{B}^{\Gamma}\left(C X, C Y \cup X, p_{X} ; \mathbb{C}\right)^{E}$ induces a fibration of spectra after applying $\mathbb{K}^{\mathrm{Top}}$ and therefore produces a long exact sequence on homotopy groups. As with [CP95, Lemma 2.5], the lemma is proved by an Eilenberg swindle. That is, we construct an endofunctor $\Sigma^{\infty}$ on $C_{r}^{*} \mathcal{B}^{\Gamma}\left(C X, C Y \cup X, p_{X} ; \mathbb{C}\right)_{C Y}$, such that $1 \oplus \Sigma^{\infty}$ is naturally equivalent to $\Sigma^{\infty}$. A category that admits such an endofunctor is called flasque. This implies that the spectrum $\mathbb{K}^{\operatorname{Top}}\left(C_{r}^{*} \mathcal{B}^{\Gamma}(C X, C Y \cup\right.$ $\left.X, p_{X} ; \mathbb{C}\right)_{C Y}$ ) has trivial homotopy groups.

Choose a continuous function $s: X \rightarrow[1, \infty)$ such that $s(x)>1$ if $x \in E$ and $s(y)=1$ for every $y \in Y$. Let $H$ be an object in $C_{r}^{*} \mathcal{B}^{\Gamma}\left(C X, C Y \cup X, p_{X} ; \mathbb{C}\right)_{C Y}$. Let $S$ be the endofunctor on $\mathcal{B}^{\Gamma}\left(C X, C Y \cup X, p_{X} ; \mathbb{C}\right)_{C Y}$ defined by $S(H)_{(x, t)}=$ $H_{(x, s(x) \cdot t)}$, where we set $H_{(x, s(x) \cdot t)}=0$ if $s(x) \cdot t \geq 1$. Since $S$ is a unitary functor, it extends to an endofunctor $\mathcal{S}$ on $C_{r}^{*} \mathcal{B}^{\Gamma}\left(C X, C Y \cup X, p_{X} ; \mathbb{C}\right)_{C Y}$. Notice that the unitary operator associated to $S, U_{H}: H \rightarrow S(H)$, is an isomorphism in $\mathcal{B}^{\Gamma}\left(C X, C Y \cup X, p_{X} ; \mathbb{C}\right)_{C Y}$. Define $\Sigma^{\infty}(H)=\bigoplus_{n \geq 1} \mathcal{S}^{n}(H)$. If $T$ is a morphism in $C_{r}^{*} \mathcal{B}^{\Gamma}\left(C X, C Y \cup X, p_{X} ; \mathbb{C}\right)_{C Y}$, then $\Sigma^{\infty}(T)$ is the diagonal operator $\bigoplus_{n \geq 1} \mathcal{S}^{n}(T)$. Since the norms of the operators $\mathcal{S}^{n}(T)$ are uniformly bounded by $\|T\|, \Sigma^{\infty}(T)$ is bounded. Since objects are zero in a neighborhood of $E, \Sigma^{\infty}$ is a well-defined endofunctor. The natural equivalence, $\eta: 1 \oplus \Sigma^{\infty} \rightarrow \Sigma^{\infty}$, is given by the diagonal unitary operator $\eta(H)=\bigoplus_{n \geq 0} U_{\mathcal{S}^{n}(H)}$.

It is worth noting that $p_{X}$-control plays an important role when $\Gamma$ is infinite. If we required continuous control everywhere, then equivariant morphisms could not have a non-zero component between $(p, s)$ and $(q, t)$ when $s \neq t$. The reason for this is that non-zero components of this type would be translated by the group to points close to $Y \times(0,1)$, contradicting control. The natural equivalence, however, requires non-zero components between such points.

The next statement follows immediately from Lemmas 3.4 and 3.5. 
Corollary 3.6. Let $\Gamma$ be a group acting on $X$ such that $Y$ is $\Gamma$-invariant. Then

$$
\mathbb{K}^{\mathrm{Top}}\left(C_{r}^{*} \mathcal{B}^{\Gamma}\left(C X, C Y \cup X, p_{X} ; \mathbb{C}\right)\right) \simeq \mathbb{K}^{\mathrm{Top}}\left(C_{r}^{*} \mathcal{B}^{\Gamma}(E \times(0,1) ; \mathbb{C})^{>0}\right) .
$$

Remark 3.7. In [HP04], Hambleton and Pedersen used the equivariant continuously controlled category $\mathcal{B}_{\Gamma}(X, Y ; R)$ to construct equivariant homology theories and to identify various versions of the assembly map. This category has a slightly different definition from the one used here. However, they agree when $\Gamma$ acts cocompactly on $E=X-Y$ with finite isotropy. It was proved in [HP04, Theorem $7.5]$ that if $E$ is a cocompact $\Gamma$-space with finite isotropy, then

$$
\pi_{i}\left(\mathbb{K}^{\mathrm{Top}}\left(C_{r}^{*} \mathcal{B}^{\Gamma}(E \times(0,1) ; \mathbb{C})^{>0}\right)\right) \cong K K_{i-1}^{\Gamma}\left(C_{0}(E) ; \mathbb{C}\right) .
$$

Furthermore, they showed that the continuously controlled assembly map

$$
\Omega \mathbb{K}^{\mathrm{Top}}\left(C_{r}^{*} \mathcal{B}^{\Gamma}(\underline{\mathrm{E} \Gamma} \times(0,1) ; \mathbb{C})^{>0}\right) \rightarrow \Omega \mathbb{K}^{\mathrm{Top}}\left(C_{r}^{*} \mathcal{B}(\bullet \times(0,1) ; \mathbb{C} \Gamma)^{>0}\right)
$$

is homotopy equivalent to the classical Baum-Connes assembly map. Corollary 3.6 implies that if $\Gamma, \underline{\mathrm{E}} \Gamma, X$ and $Y$ satisfy the conditions of the main theorem, then

$$
\pi_{i}\left(\mathbb{K}^{\mathrm{Top}}\left(C_{r}^{*} \mathcal{B}^{\Gamma}\left(C X, C Y \cup X, p_{X} ; \mathbb{C}\right)\right)\right) \cong K K_{i-1}^{\Gamma}\left(C_{0}(\underline{\mathrm{E}} \Gamma) ; \mathbb{C}\right) .
$$

Combining this with the following theorem shows that the Baum-Connes assembly map is equivalent to

$$
\Omega \mathbb{K}^{\mathrm{Top}}\left(C_{r}^{*} \mathcal{B}^{\Gamma}\left(C X, C Y \cup X, p_{X} ; \mathbb{C}\right)\right) \rightarrow \Omega \mathbb{K}^{\mathrm{Top}}\left(C_{r}^{*} \mathcal{B}^{\Gamma}\left(\Sigma X, \Sigma Y, p_{X} ; \mathbb{C}\right)\right),
$$

which is induced by collapsing $X$ to a point.

Theorem 3.8. Assume that $\Gamma, \underline{\mathrm{E}} \Gamma, X$ and $Y$ satisfy the conditions of the main theorem. Then $\Omega \mathbb{K}^{\mathrm{Top}}\left(C_{r}^{*} \mathcal{B}^{\Gamma}\left(\Sigma X, \Sigma Y, p_{X} ; \mathbb{C}\right)\right) \simeq \mathbb{K}^{\mathrm{Top}}\left(C_{r}^{*} \Gamma\right)$.

Proof. The assumption that the compact subsets of $\underline{\mathrm{E}} \Gamma$ shrink at infinity implies

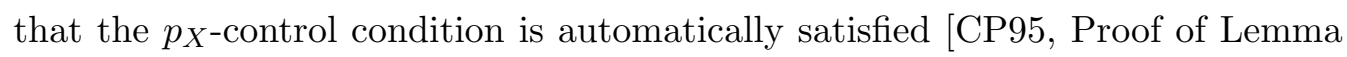
2.3]. Thus, $C_{r}^{*} \mathcal{B}^{\Gamma}\left(\Sigma X, \Sigma Y, p_{X} ; \mathbb{C}\right)=C_{r}^{*} \mathcal{B}^{\Gamma}(\Sigma E \Gamma,\{0,1\} ; \mathbb{C})$. The quotient map $q: \Sigma \underline{E} \Gamma \rightarrow \Sigma(\underline{\mathrm{E}} \Gamma / \Gamma)$, which is eventually continuous, induces an equivalence of categories

$$
\mathcal{B}^{\Gamma}(\Sigma \underline{E} \Gamma,\{0,1\} ; \mathbb{C}) \rightarrow \mathcal{B}(\Sigma(\underline{\mathrm{E}} \Gamma / \Gamma),\{0,1\} ; \mathbb{C} \Gamma) .
$$

To show this, we define the inverse functor $V$ as follows. Let $H$ be an object of $\mathcal{B}(\Sigma(\underline{\mathrm{E}} \Gamma / \Gamma),\{0,1\} ; \mathbb{C} \Gamma)$. Recall that for every $z \in \Sigma(\underline{\mathrm{E}} \Gamma / \Gamma), H_{z}$ is a finitely generated free $\mathbb{C} \Gamma$-module. Therefore $H_{z}=\bigoplus_{g \in \Gamma} H_{(z, g)}$, where $H_{(z, g)}$ is a complex vector space whose dimension is equal to the $\mathbb{C} \Gamma$-dimension of $H_{z}$. Define 
$V(H)_{x}=\bigoplus_{g \in \Gamma_{x}} H_{(q(x), g)}$, where $\Gamma_{x}$ denotes the stabilizer subgroup of $x$. The fact that $V$ is a functor depends on the assumption that $\mathrm{E} \Gamma$ is a finite $\Gamma-\mathrm{CW}$ complex. Furthermore, $V$ is a unitary functor since there is a corresponding unitary operator for each object. Thus, the equivalence extends to its $C_{r}^{*}$-counterpart,

$$
C_{r}^{*} \mathcal{B}^{\Gamma}\left(\Sigma X, \Sigma Y, p_{X} ; \mathbb{C}\right) \cong C_{r}^{*} \mathcal{B}(\Sigma(\underline{E} \Gamma / \Gamma),\{0,1\} ; \mathbb{C} \Gamma) .
$$

By Proposition $3.2, C_{r}^{*} \mathcal{B}(\Sigma(\underline{\mathrm{E}} \Gamma / \Gamma),\{0,1\} ; \mathbb{C} \Gamma) \cong C_{r}^{*} \mathcal{B}([0,1],\{0,1\} ; \mathbb{C} \Gamma)$. To finish the proof, consider the commutative diagram

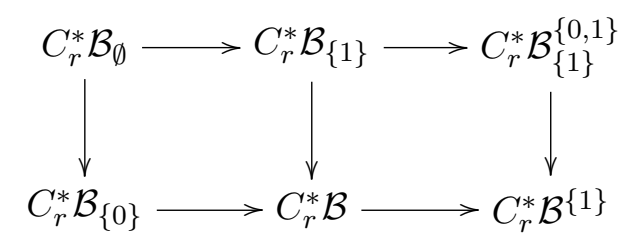

in which $\mathcal{B}=\mathcal{B}([0,1],\{0,1\} ; \mathbb{C} \Gamma)$. Notice that the rightmost vertical map is an equivalence of categories and that both $C_{r}^{*} \mathcal{B}_{\{0\}}$ and $C_{r}^{*} \mathcal{B}_{\{1\}}$ are flasque. Each row of the diagram is a Karoubi filtration inducing a fibration of spectra after applying $\mathbb{K}^{\text {Top }}$ and hence a long exact sequence of homotopy groups. Therefore, $\mathbb{K}^{\mathrm{Top}}\left(C_{r}^{*} \mathcal{B}\right) \simeq \Sigma \mathbb{K}^{\mathrm{Top}}\left(C_{r}^{*} \mathcal{B}_{\emptyset}\right)$. The local finiteness of objects implies $C_{r}^{*} \mathcal{B}_{\emptyset} \cong$ $C_{r}^{*} \mathcal{B}(\bullet, \emptyset ; \mathbb{C} \Gamma)$. Thus, $\mathbb{K}^{\operatorname{Top}}\left(C_{r}^{*} \mathcal{B}\right) \simeq \Sigma \mathbb{K}^{\operatorname{Top}}\left(C_{r}^{*} \Gamma\right)$.

3.2. The Conner Conjecture and Steenrod Homology. Recall that a reduced Steenrod homology theory, $h$, is a functor from the category of compact metrizable spaces and continuous maps to the category of graded abelian groups satisfying

(i) $h$ is homotopy invariant;

(ii) $h(\bullet)=0$;

(iii) given any closed subset $A$ of $X$, there is a natural transformation, $\partial$ : $h_{n}(X / A) \rightarrow h_{n-1}(A)$, fitting into a long exact sequence

$$
\cdots \rightarrow h_{n}(A) \rightarrow h_{n}(X) \rightarrow h_{n}(X / A) \rightarrow h_{n-1}(A) \rightarrow \cdots ;
$$

(iv) if $\bigvee X_{i}$ denotes a compact metric space that is a countable union of metric spaces along a single point, then the projection maps $p_{i}: \bigvee X_{i} \rightarrow X_{i}$ induce an isomorphism $h_{*}\left(\bigvee X_{i}\right) \rightarrow \prod h_{*}\left(X_{i}\right)$.

Given any generalized homology theory, there is a unique Steenrod homology extension. Existence of such extensions was proved by Kahn, Kaminker and 
Schochet, and Edwards and Hastings [KKS77, EH76]. Uniqueness was proved by Milnor [Mil63].

A functor $k$ from the category of compact metrizable spaces to the category of spectra is called a reduced Steenrod functor if it satisfies the following conditions.

(i) The spectrum $k(C X)$ is contractible.

(ii) If $A \subset X$ is closed, then $k(A) \rightarrow k(X) \rightarrow k(X / A)$ is a fibration (up to natural weak homotopy equivalence).

(iii) The projection maps $p_{i}: \bigvee X_{i} \rightarrow X_{i}$ induce a weak homotopy equivalence $k\left(\bigvee X_{i}\right) \rightarrow \prod k\left(X_{i}\right)$.

Theorem 3.9. [HPR97, Proposition 12.1] Let $H$ be a discrete group. The functor

$$
\mathbb{K}^{\mathrm{Top}}\left(C_{r}^{*} \mathcal{B}(C(-),-; \mathbb{C} H)\right)
$$

is a reduced Steenrod functor whose value on $S^{0}$ is $\Sigma \mathbb{K}^{\mathrm{Top}}\left(C_{r}^{*} H\right)$.

Given the work done thus far, it is easy to see that the proof of [CP95, Theorem $2.13]$ extends to give the following result. This proposition plays a key role in the proof of Theorem 4.4 .

Proposition 3.10. Let $H$ be a discrete group, $X$ a compact metrizable space, and $Y$ a closed nowhere dense subset. If the reduced Steenrod homology of $X$ (from Theorem 3.9) is trivial, then

$$
\mathbb{K}^{\mathrm{Top}}\left(C_{r}^{*} \mathcal{B}\left(C X, C Y \cup X, p_{X} ; \mathbb{C} H\right)\right) \simeq \mathbb{K}^{\mathrm{Top}}\left(C_{r}^{*} \mathcal{B}\left(\Sigma X, \Sigma Y, p_{X} ; \mathbb{C} H\right)\right) .
$$

In the proof of Theorem 4.4 we will be considering spaces with a finite group action. In order to use Proposition 3.10 on the corresponding quotient spaces, we need to know that the reduced Steenrod homology (from Theorem 3.9) of the quotient of a contractible compact metrizable space by a finite group is trivial. The first step toward proving this is [Bre72, Theorem III.7.12], which states that if $G$ is a finite group acting on a compact Hausdorff space $X$ that has trivial reduced Čech cohomology, then the reduced Čech cohomology of $X / G$ is also trivial. This is a special case of the Conner Conjecture which states that if a compact Lie group $G$ acts on a space $X$, where $X$ is either paracompact of finite cohomological dimension with finitely many orbit types or compact Hausdorff, then $X / G$ has trivial reduced Čech cohomology if $X$ does [Con60]. The Conner Conjecture was proved by Oliver [Oli76]. The final step is the following theorem. 
Theorem 3.11. [Ros04, Theorem 5.2] If the reduced Čech cohomology of a compact metrizable space $X$ is trivial, then the reduced Steenrod homology (from Theorem 3.9) of $X$ is also trivial.

Proof. Let $A$ be an abelian group, let $H_{q}(X ; A)$ denote the ordinary unreduced Steenrod homology of $X$ with coefficients in $A$, let $\widetilde{H}_{q}(X ; A)$ denote the ordinary reduced Steenrod homology of $X$ with coefficients in $A$, and let $\check{H}^{q}(X ; A)$ denote the unreduced Čech cohomology of $X$ with coefficients in $A$. Note that $H_{*}$ is related to $\check{H}^{*}$ by a split exact sequence

$$
0 \rightarrow \operatorname{Ext}\left(\check{H}^{q+1}(X), A\right) \rightarrow H_{q}(X ; A) \rightarrow \operatorname{Hom}\left(\check{H}^{q}(X), A\right) \rightarrow 0
$$

[Mil63, Lemma 5]. Since the reduced Čech cohomology of $X$ is trivial, this implies that $\widetilde{H}_{q}(X ; A)=0$ for all $q$.

There is a spectral sequence converging to $\pi_{*}\left(\mathbb{K}^{\operatorname{Top}}\left(C_{r}^{*} \mathcal{B}(C X, X ; \mathbb{C} H)\right)\right)$ with

$$
E_{p, q}^{2}=\widetilde{H}_{p}\left(X ; \pi_{q}\left(\mathbb{K}^{\mathrm{Top}}\left(C_{r}^{*} \mathcal{B}\left(C\left(S^{0}\right), S^{0} ; \mathbb{C} H\right)\right)\right)\right)=\widetilde{H}_{p}\left(X ; K_{q-1}\left(C_{r}^{*} H\right)\right)
$$

[EH76, 8.5.5]. Each term of the spectral sequence is zero, which completes the proof.

As mentioned above, the following corollary will be used in the proof of Theorem 4.4.

Corollary 3.12. Let $G$ be a finite group acting on a compact metrizable space $X$. If the reduced Čech cohomology of $X$ is trivial, then the reduced Steenrod homology (from Theorem 3.9) of $X / G$ is trivial.

\section{The Main Theorem}

Theorem 4.1. Let $\Gamma$ be a discrete group, and let $\underline{\mathrm{E}} \Gamma$ be a finite $\Gamma-C W$ model for the universal space for $\Gamma$-actions with finite isotropy. Assume that $\underline{\mathrm{E}} \Gamma$ admits a compactification, $X$, (i.e., $X$ is compact and $\underline{\mathrm{E} \Gamma}$ is an open dense subset) such that

1. the $\Gamma$-action extends to $X$;

2. $X$ is metrizable;

3. $X^{G}$ is contractible for every finite subgroup $G$ of $\Gamma$;

4. $\underline{\mathrm{E}}^{G}$ is dense in $X^{G}$ for every finite subgroup $G$ of $\Gamma$; 
5. compact subsets of $\underline{\mathrm{E} \Gamma}$ become small near $Y=X-\underline{\mathrm{E}} \Gamma$. That is, for every compact subset $K \subset \mathrm{E} \Gamma$ and for every neighborhood $U \subset X$ of $y \in Y$, there exists a neighborhood $V \subset X$ of $y$ such that $g \in \Gamma$ and $g K \cap V \neq \emptyset$ implies $g K \subset U$.

Then the Baum-Connes map, $K K_{i}^{\Gamma}\left(C_{0}(\underline{\mathrm{E}} \Gamma) ; \mathbb{C}\right) \rightarrow K_{i}\left(C_{r}^{*} \Gamma\right)$, is a split injection.

There are many classes of groups satisfying the conditions of the main theorem. Crystallographic groups, which are discrete groups that act cocompactly on Euclidean $n$-space by isometries, satisfy these conditions. The desired compactification is obtained by adding an $(n-1)$-sphere at infinity. Gromov's word hyperbolic groups also satisfy the conditions by taking a certain compactification of the Rips complex [RS05]. Much has already been proved about word hyperbolic groups. Higson proved that the Baum-Connes map is a split injection for countable groups that admit an amenable action on a compact Hausdorff space [Hig00], and a word hyperbolic group acts amenably on its Gromov boundary [Ad94]. Later, Mineyev and Yu [MY02] showed that the Baum-Connes assembly map is in fact an isomorphism for these groups.

4.1. The Proof. As was seen in Section 3.1, the map

$$
\Omega \mathbb{K}^{\mathrm{Top}}\left(C_{r}^{*} \mathcal{B}^{\Gamma}\left(C X, C Y \cup X, p_{X} ; \mathbb{C}\right)\right) \rightarrow \Omega \mathbb{K}^{\mathrm{Top}}\left(C_{r}^{*} \mathcal{B}^{\Gamma}\left(\Sigma X, \Sigma Y, p_{X} ; \mathbb{C}\right)\right)
$$

produces the Baum-Connes map after taking homotopy groups. It fits in to the following commutative diagram, in which $\mathcal{F}$ denotes the family of finite subgroups of $\Gamma, \mathcal{B}(C X)=\mathcal{B}\left(C X, C Y \cup X, p_{X} ; \mathbb{C}\right)$ and $\mathcal{B}(\Sigma X)=\mathcal{B}^{\Gamma}\left(\Sigma X, \Sigma Y, p_{X} ; \mathbb{C}\right)$.

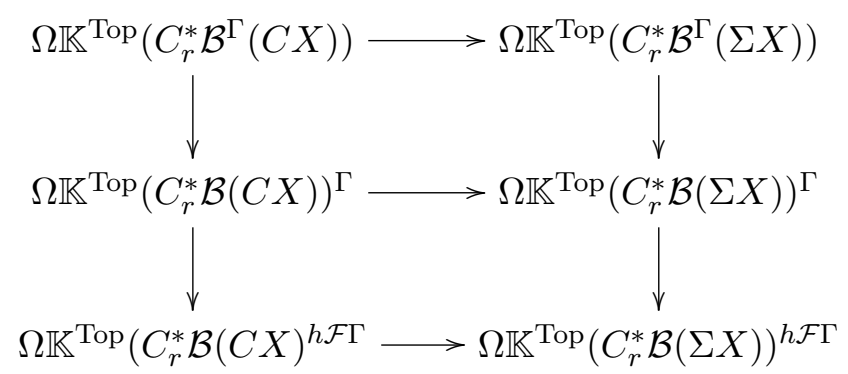

The main theorem is proved by showing that the composition

$$
\Omega \mathbb{K}^{\mathrm{Top}}\left(C_{r}^{*} \mathcal{B}^{\Gamma}(C X)\right) \rightarrow \Omega \mathbb{K}^{\mathrm{Top}}\left(C_{r}^{*} \mathcal{B}(C X)\right)^{\Gamma} \rightarrow \Omega \mathbb{K}^{\mathrm{Top}}\left(C_{r}^{*} \mathcal{B}(C X)\right)^{h \mathcal{F} \Gamma}
$$


and the map

$$
\Omega \mathbb{K}^{\mathrm{Top}}\left(C_{r}^{*} \mathcal{B}(C X)\right)^{h \mathcal{F} \Gamma} \rightarrow \Omega \mathbb{K}^{\mathrm{Top}}\left(C_{r}^{*} \mathcal{B}(\Sigma X)\right)^{h \mathcal{F} \Gamma}
$$

are weak homotopy equivalences. In this section the first of these equivalences is handled. The second is proved in the subsequent section.

For the remainder of this paper, assume that $\Gamma, \underline{\mathrm{E}} \Gamma, X$, and $Y$ satisfy the conditions of the main theorem.

Theorem 4.2. The spectrum $\mathbb{K}^{\mathrm{Top}}\left(C_{r}^{*} \mathcal{B}^{\Gamma}(\underline{\mathrm{E}} \Gamma \times(0,1) ; \mathbb{C})^{>0}\right)$ is weakly homotopy equivalent to $\mathbb{K}^{\operatorname{Top}}\left(C_{r}^{*} \mathcal{B}(\underline{\mathrm{E}} \Gamma \times(0,1) ; \mathbb{C})^{>0}\right)^{h \mathcal{F} \Gamma}$.

Proof. Note that $\mathrm{E} \Gamma$ is assumed to be a finite $\Gamma-\mathrm{CW}$ complex, and proceed by induction on the $\Gamma$-cells in $\underline{E} \Gamma$. Begin with the discrete space $\Gamma / H$ for some $H \in \mathcal{F}$. The control condition implies that the components of a morphism near $\Gamma / H \times 1$ must be zero between points with different $\Gamma / H$ entries. Since we are taking germs at $\Gamma / H \times 1$, the category $\mathcal{B}(\Gamma / H \times(0,1) ; \mathbb{C})^{>0}$ is equivalent to the product category $\prod_{\Gamma / H} \mathcal{B}((0,1) ; \mathbb{C})^{>0}$. Thus:

$$
C_{r}^{*} \mathcal{B}(\Gamma / H \times(0,1) ; \mathbb{C})^{>0} \cong \bigoplus_{\Gamma / H} C_{r}^{*} \mathcal{B}((0,1) ; \mathbb{C})^{>0}
$$

The $\Gamma$-action on these categories is the one described in Lemma 2.4. Therefore, using Lemma 2.2,

$$
\begin{aligned}
\left(C_{r}^{*} \mathcal{B}(\Gamma / H \times(0,1) ; \mathbb{C})^{>0}\right)^{\Gamma} & \cong\left(\bigoplus_{\Gamma / H} C_{r}^{*} \mathcal{B}((0,1) ; \mathbb{C})^{>0}\right)^{\Gamma} \\
& \cong \quad\left(C_{r}^{*} \mathcal{B}((0,1) ; \mathbb{C})^{>0}\right)^{H} \\
& \cong \quad C_{r}^{*} \mathcal{B}^{H}((0,1) ; \mathbb{C})^{>0} \\
& \cong \quad C_{r}^{*}\left(\left(\prod_{\Gamma / H} \mathcal{B}((0,1) ; \mathbb{C})^{>0}\right)^{\Gamma}\right) \\
& \cong \quad C_{r}^{*} \mathcal{B}^{\Gamma}(\Gamma / H \times(0,1) ; \mathbb{C})^{>0} .
\end{aligned}
$$

Taking fixed sets commutes with applying $\mathbb{K}^{\text {Top }}$, therefore:

$$
\mathbb{K}^{\mathrm{Top}}\left(C_{r}^{*} \mathcal{B}^{\Gamma}(\Gamma / H \times(0,1) ; \mathbb{C})^{>0}\right) \cong \mathbb{K}^{\mathrm{Top}}\left(C_{r}^{*} \mathcal{B}(\Gamma / H \times(0,1) ; \mathbb{C})^{>0}\right)^{\Gamma}
$$

We now need to show that $\mathbb{K}^{\operatorname{Top}}\left(C_{r}^{*} \mathcal{B}(\Gamma / H \times(0,1) ; \mathbb{C})^{>0}\right)^{\Gamma}$ is weakly homotopy equivalent to $\mathbb{K}^{\operatorname{Top}}\left(C_{r}^{*} \mathcal{B}(\Gamma / H \times(0,1) ; \mathbb{C})^{>0}\right)^{h \mathcal{F} \Gamma}$. 
The projection maps induce a map

$$
\mathbb{K}^{\operatorname{Top}}\left(\bigoplus_{\Gamma / H} C_{r}^{*} \mathcal{B}((0,1) ; \mathbb{C})^{>0}\right) \rightarrow \prod_{\Gamma / H} \mathbb{K}^{\mathrm{Top}}\left(C_{r}^{*} \mathcal{B}((0,1) ; \mathbb{C})^{>0}\right)
$$

that is $\Gamma$-equivariant and a weak homotopy equivalence (see Section 2.2). Consider the following commutative diagram:

$$
\begin{aligned}
& \mathbb{K}^{\operatorname{Top}}\left(\bigoplus_{\Gamma / H} C_{r}^{*} \mathcal{B}((0,1) ; \mathbb{C})^{>0}\right)^{\Gamma} \stackrel{b}{\longrightarrow}\left(\prod_{\Gamma / H} \mathbb{K}^{\operatorname{Top}}\left(C_{r}^{*} \mathcal{B}((0,1) ; \mathbb{C})^{>0}\right)\right)^{\Gamma} \\
& \mathbb{K}^{\mathrm{Top}}\left(\bigoplus_{\Gamma / H} C_{r}^{*} \mathcal{B}((0,1) ; \mathbb{C})^{>0}\right)^{h \mathcal{F} \Gamma} \stackrel{d}{\longrightarrow}\left(\prod_{\Gamma / H} \mathbb{K}^{\operatorname{Top}}\left(C_{r}^{*} \mathcal{B}((0,1) ; \mathbb{C})^{>0}\right)\right)^{h \mathcal{F} \Gamma}
\end{aligned}
$$

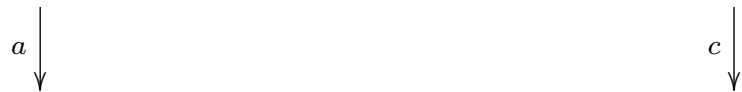

We want to show that $a$ is a weak homotopy equivalence. Let $G \leq \Gamma$ be given, and choose representatives $\gamma_{j}$, so that $G \backslash \Gamma / H=\left\{G \gamma_{j} H\right\}$. Then,

$$
\begin{aligned}
\mathbb{K}^{\operatorname{Top}}\left(\bigoplus_{\Gamma / H} C_{r}^{*} \mathcal{B}((0,1) ; \mathbb{C})^{>0}\right)^{G} & \cong \mathbb{K}^{\operatorname{Top}}\left(\left(\bigoplus_{\Gamma / H} C_{r}^{*} \mathcal{B}((0,1) ; \mathbb{C})^{>0}\right)^{G}\right) \\
& \cong \mathbb{K}^{\operatorname{Top}}\left(\bigoplus_{G \backslash \Gamma / H} C_{r}^{*} \mathcal{B}^{\gamma_{j}^{-1} G \gamma_{j} \cap H}((0,1) ; \mathbb{C})^{>0}\right) \\
& \simeq \prod_{G \backslash \Gamma / H} \mathbb{K}^{\mathrm{Top}}\left(C_{r}^{*} \mathcal{B}^{\gamma_{j}^{-1} G \gamma_{j} \cap H}((0,1) ; \mathbb{C})^{>0}\right) \\
& \simeq \prod_{G \backslash \Gamma / H} \mathbb{K}^{\mathrm{Top}}\left(C_{r}^{*} \mathcal{B}((0,1) ; \mathbb{C})^{>0}\right)^{\gamma_{j}^{-1} G \gamma_{j} \cap H} \\
& \cong\left(\prod_{\Gamma / H} \mathbb{K}^{\operatorname{Top}}\left(C_{r}^{*} \mathcal{B}((0,1) ; \mathbb{C})^{>0}\right)^{G}\right.
\end{aligned}
$$

by Lemma 2.2 and the fact that $\mathbb{K}^{\text {Top }}$ commutes with taking fixed sets. The case $G=\Gamma$ proves that $b$ is a weak homotopy equivalence. By Lemma $2.3, d$ is a weak homotopy equivalence, and finally, $c$ is a weak homotopy equivalence by Lemma 2.4. This completes the base case of the induction.

Now assume that the theorem holds for $N$ and that $E$ is obtained from $N$ by attaching an $n$-cell, $\Gamma / K \times \mathbb{D}^{n}$, for some $K \in \mathcal{F}$. Since $C_{r}^{*} \mathcal{B}^{\Gamma}(E \times(0,1) ; \mathbb{C})_{N \times 1}^{>0}$ is equivalent to $C_{r}^{*} \mathcal{B}^{\Gamma}(N \times(0,1) ; \mathbb{C})^{>0}$,

$C_{r}^{*} \mathcal{B}^{\Gamma}(N \times(0,1) ; \mathbb{C})^{>0} \rightarrow C_{r}^{*} \mathcal{B}^{\Gamma}(E \times(0,1) ; \mathbb{C})^{>0} \rightarrow C_{r}^{*} \mathcal{B}^{\Gamma}(E \times(0,1) ; \mathbb{C})^{(E-N) \times 1}$ 
is a Karoubi filtration, which induces a fibration of spectra after applying $\mathbb{K}^{\mathrm{Top}}$. The same statement is true if $\Gamma$ is replaced by a finite subgroup $G$. Let $\mathcal{A}=$ $\mathcal{B}(N \times(0,1) ; \mathbb{C})^{>0}, \mathcal{B}=\mathcal{B}(E \times(0,1) ; \mathbb{C})^{>0}$, and $\mathcal{C}=\mathcal{B}(E \times(0,1) ; \mathbb{C})^{(E-N) \times 1}$, and consider the following commutative diagram:

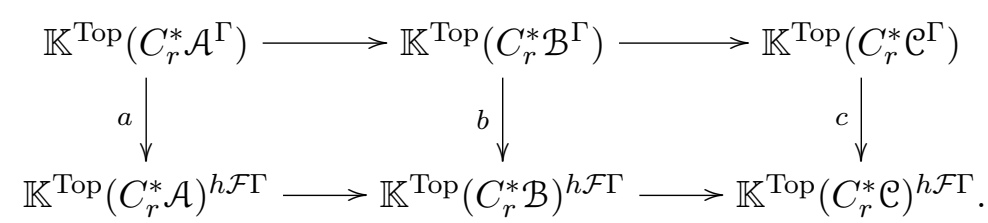

By the induction hypothesis, $a$ is a weak homotopy equivalence. To complete the proof, we need to show that $b$ is a weak homotopy equivalence. The top row in the diagram is a fibration of spectra. By the Five Lemma, it suffices to prove that $c$ is a weak homotopy equivalence once we know that the bottom row is also a fibration of spectra. As mentioned above, $C_{r}^{*} \mathcal{A}^{G} \rightarrow C_{r}^{*} \mathcal{B}^{G} \rightarrow C_{r}^{*} \mathrm{e}^{G}$ induces a fibration after applying $\mathbb{K}^{\text {Top }}$, for every $G \in \mathcal{F}$. Thus, by Lemma 2.2,

$$
\mathbb{K}^{\mathrm{Top}}\left(C_{r}^{*} \mathcal{A}\right)^{G} \rightarrow \mathbb{K}^{\mathrm{Top}}\left(C_{r}^{*} \mathcal{B}\right)^{G} \rightarrow \mathbb{K}^{\mathrm{Top}}\left(C_{r}^{*} \mathcal{C}\right)^{G}
$$

is a fibration for every $G \in \mathcal{F}$. Let $F$ denote the homotopy fiber of $\mathbb{K}^{\text {Top }}\left(C_{r}^{*} \mathcal{B}\right) \rightarrow$ $\mathbb{K}^{\mathrm{Top}}\left(C_{r}^{*} \mathrm{C}\right)$. Taking homotopy fixed sets and taking homotopy fibers are commutative operations since both are inverse limits. This implies that $F^{h \mathcal{F} \Gamma} \rightarrow$ $\mathbb{K}^{\operatorname{Top}}\left(C_{r}^{*} \mathcal{B}\right)^{h \mathcal{F} \Gamma} \rightarrow \mathbb{K}^{\operatorname{Top}}\left(C_{r}^{*} \mathcal{C}\right)^{h \mathcal{F} \Gamma}$ is a fibration of spectra. Taking fixed sets is also an inverse limit, so it too commutes with homotopy fibers. Therefore, $F^{G} \rightarrow \mathbb{K}^{\operatorname{Top}}\left(C_{r}^{*} \mathcal{B}\right)^{G} \rightarrow \mathbb{K}^{\operatorname{Top}}\left(C_{r}^{*} \mathcal{C}\right)^{G}$ is a fibration for every $G \in \mathcal{F}$. Thus, $F^{G} \simeq \mathbb{K}^{\operatorname{Top}}\left(C_{r}^{*} \mathcal{A}\right)^{G}$ for every $G \in \mathcal{F}$. By Lemma $2.3, F^{h \mathcal{F} \Gamma} \simeq \mathbb{K}^{\operatorname{Top}}\left(C_{r}^{*} \mathcal{A}\right)^{h \mathcal{F} \Gamma}$. Therefore, the bottom row in the above diagram is a fibration. Now, since $E-N=\Gamma / K \times e^{n}$ for some $K \in \mathcal{F}$, where $e^{n}$ is an open $n$-cell, the category $\mathcal{B}(E \times(0,1) ; \mathbb{C})^{(E-N) \times 1}$ is equivalent to the product category $\prod_{\Gamma / K} \mathcal{B}\left(e^{n} \times\right.$ $(0,1) ; \mathbb{C})^{>0}$. Therefore, the proof that $c$ is a weak homotopy equivalence is analogous to the start of the induction.

Corollary 4.3. The spectrum $\mathbb{K}^{\mathrm{Top}}\left(C_{r}^{*} \mathcal{B}^{\Gamma}\left(C X, C Y \cup X, p_{X} ; \mathbb{C}\right)\right)$ is weakly homotopy equivalent to $\mathbb{K}^{\mathrm{Top}}\left(C_{r}^{*} \mathcal{B}\left(C X, C Y \cup X, p_{X} ; \mathbb{C}\right)\right)^{h \mathcal{F} \Gamma}$. 
Proof.

$$
\begin{aligned}
\mathbb{K}^{\mathrm{Top}}\left(C_{r}^{*} \mathcal{B}^{\Gamma}\left(C X, C Y \cup X, p_{X} ; \mathbb{C}\right)\right) & \simeq \mathbb{K}^{\mathrm{Top}}\left(C_{r}^{*} \mathcal{B}^{\Gamma}(\underline{\mathrm{E} \Gamma} \times(0,1) ; \mathbb{C})^{>0}\right) \\
& \simeq \mathbb{K}^{\mathrm{Top}}\left(C_{r}^{*} \mathcal{B}(\underline{\mathrm{E} \Gamma} \times(0,1) ; \mathbb{C})^{>0}\right)^{h \mathcal{F} \Gamma} \\
& \simeq \mathbb{K}^{\mathrm{Top}}\left(C_{r}^{*} \mathcal{B}\left(C X, C Y \cup X, p_{X} ; \mathbb{C}\right)\right)^{h \mathcal{F} \Gamma}
\end{aligned}
$$

The first equivalence follows from Lemmas 3.4 and 3.5. The second equivalence follows from Theorem 4.2. To establish the third equivalence, notice that Lemmas 3.4 and 3.5 hold when $\Gamma$ is replaced by any of its finite subgroups. Thus, Lemma 2.2 implies that $\mathbb{K}^{\text {Top }}\left(C_{r}^{*} \mathcal{B}\left(C X, C Y \cup X, p_{X} ; \mathbb{C}\right)\right)^{G} \simeq$ $\mathbb{K}^{\mathrm{Top}}\left(C_{r}^{*} \mathcal{B}(\underline{\mathrm{E}} \Gamma \times(0,1) ; \mathbb{C})^{>0}\right)^{G}$ for every $G \in \mathcal{F}$. Now Lemma 2.3 finishes the proof.

4.2. Filtering by Conjugacy Classes of Fixed Sets. In this section we complete the proof of the main theorem by showing that

$$
\Omega \mathbb{K}^{\operatorname{Top}}\left(C_{r}^{*} \mathcal{B}\left(C X, C Y \cup X, p_{X} ; \mathbb{C}\right)\right)^{h \mathcal{F} \Gamma} \simeq \Omega \mathbb{K}^{\operatorname{Top}}\left(C_{r}^{*} \mathcal{B}\left(\Sigma X, \Sigma Y, p_{X} ; \mathbb{C}\right)\right)^{h \mathcal{F} \Gamma} .
$$

By Lemmas 2.2 and 2.3, it suffices to prove the following.

Theorem 4.4. For every $G \in \mathcal{F}$,

$$
\mathbb{K}^{\mathrm{Top}}\left(C_{r}^{*} \mathcal{B}^{G}\left(C X, C Y \cup X, p_{X} ; \mathbb{C}\right)\right) \simeq \mathbb{K}^{\mathrm{Top}}\left(C_{r}^{*} \mathcal{B}^{G}\left(\Sigma X, \Sigma Y, p_{X} ; \mathbb{C}\right)\right) .
$$

Given $G \in \mathcal{F}$, consider the subgroup lattice for $G$, and let $H \leq G$. Define the distance from $G$ to $H$, $\operatorname{dist}(H)$, to be the maximum number of steps needed to reach $H$ from $G$ on the subgroup lattice. Notice that $\operatorname{dist}\left(g \mathrm{Hg}^{-1}\right)=\operatorname{dist}(H)$ for each $g \in G$. Let $n=\operatorname{dist}(1)$, and let $l_{i}$ be the number of conjugacy classes of subgroups with distance $i$ from $G$. For each $i, 1 \leq i \leq n-1$, choose a representative $H_{i, j}, 1 \leq j \leq l_{i}$, for each of the conjugacy classes with distance $i$ from $G$. Order the $H_{i, j}$ 's using the dictionary order on the indexing set. Now re-index the sequence of subgroups according to the ordering so that

$$
H_{1}=H_{1,1} \quad, \quad H_{2}=H_{1,2} \quad, \ldots, \quad H_{m}=H_{n-1, l_{n-1}} .
$$

For convenience define $H_{0}=G$ and $H_{m+1}=1$.

For each $j, 0 \leq j \leq m+1$, define $C_{j}=\bigcup_{g \in G} X^{g H_{j} g^{-1}}$, which will often be referred to as a conjugacy class of fixed sets. For each $k, 0 \leq k \leq m+1$, define $Z_{k}=\bigcup_{0 \leq j \leq k} C_{j}$. Also define $Y_{k}=Z_{k} \cap Y$. Since it is possible that $Y_{k}=\emptyset$, we define $C(\emptyset)=\{0\}$ and $\Sigma(\emptyset)=\{0,1\}$ for the convenience of notation. 
Notice that $Z_{k}$ is contractible for every $k, 0 \leq k \leq m+1$. Also notice that since $g X^{H}=X^{g H g^{-1}}$, each of the conjugacy classes of fixed sets is $G$-invariant. Therefore, $Z_{k}$ is $G$-invariant for every $k, 0 \leq k \leq m+1$.

To simplify the notation, let

$$
\begin{aligned}
& \mathcal{B}_{\mathbb{C}}^{G}\left(C\left(Z_{k}\right)\right)=\mathcal{B}^{G}\left(C\left(Z_{k}\right), C\left(Y_{k}\right) \cup Z_{k}, p_{Z_{k}} ; \mathbb{C}\right) ; \\
& \mathcal{B}_{\mathbb{C}}^{G}\left(\Sigma\left(Z_{k}\right)\right)=\mathcal{B}^{G}\left(\Sigma\left(Z_{k}\right), \Sigma\left(Y_{k}\right), p_{Z_{k}} ; \mathbb{C}\right) ; \\
& \mathcal{B}_{\mathbb{C}}^{G}\left(C\left(Z_{k}\right) ; \mathbb{C}\right)_{Z_{k-1}}=\mathcal{B}_{\mathbb{C}}^{G}\left(C\left(Z_{k}\right)\right)_{C\left(Y_{k-1}\right) \cup Z_{k-1}} ; \\
& \mathcal{B}_{\mathbb{C}}^{G}\left(\Sigma\left(Z_{k}\right)\right)_{Z_{k-1}}=\mathcal{B}_{\mathbb{C}}^{G}\left(\Sigma\left(Z_{k}\right)\right)_{\Sigma\left(Y_{k-1}\right)} ; \\
& \mathcal{B}_{\mathbb{C}}^{G}\left(C\left(Z_{k}\right)\right)^{>Z_{k-1}}=\mathcal{B}_{\mathbb{C}}^{G}\left(C\left(Z_{k}\right)\right)^{\left(C\left(Y_{k}\right) \cup Z_{k}\right)-\left(C\left(Y_{k-1}\right) \cup Z_{k-1}\right)} ; \\
& \mathcal{B}_{\mathbb{C}}^{G}\left(\Sigma\left(Z_{k}\right)\right)^{>Z_{k-1}}=\mathcal{B}_{\mathbb{C}}^{G}\left(\Sigma\left(Z_{k}\right)\right)^{\Sigma\left(Y_{k}\right)-\Sigma\left(Y_{k-1}\right)} .
\end{aligned}
$$

Theorem 4.4 is proved by induction on the chain

$$
X^{G}=Z_{0} \subseteq Z_{1} \subseteq \cdots \subseteq Z_{m} \subseteq Z_{m+1}=X .
$$

As in [Ros04], this is accomplished with the following three lemmas.

Lemma 4.5. Let $1 \leq k \leq m+1$. Then:

i. $C_{r}^{*} \mathcal{B}_{\mathbb{C}}^{G}\left(C\left(Z_{k}\right)\right)_{Z_{k-1}} \cong C_{r}^{*} \mathcal{B}_{\mathbb{C}}^{G}\left(C\left(Z_{k-1}\right)\right)$

ii. $C_{r}^{*} \mathcal{B}_{\mathbb{C}}^{G}\left(\Sigma\left(Z_{k}\right)\right)_{Z_{k-1}} \cong C_{r}^{*} \mathcal{B}_{\mathbb{C}}^{G}\left(\Sigma\left(Z_{k-1}\right)\right)$

Proof. The proof of this lemma is given in [Ros06, Lemma 3.3], and is included here for the reader's convenience. Consider part (i). The equivalence is proved by constructing an equivariant function $f$, from $C\left(Z_{k}\right)$ into itself, that is not quite an eventually continuous map, but is good enough to induce a functor from $C_{r}^{*} \mathcal{B}_{\mathbb{C}}^{G}\left(C\left(Z_{k}\right)\right)_{Z_{k-1}}$ to $C_{r}^{*} \mathcal{B}_{\mathbb{C}}^{G}\left(C\left(Z_{k-1}\right)\right)$ that is an inverse to the inclusion functor up to natural equivalence. The only difficulty in defining such a function appears when there are sequences consisting of points outside of $C\left(Z_{k-1}\right)$ that converge to points in $C\left(Y_{k-1}\right) \cup Z_{k-1}$. If this does not happen, then every point not in $C\left(Z_{k-1}\right)$ is greater than a fixed distance away from $C\left(Y_{k-1}\right) \cup Z_{k-1}$. Since objects are zero on a neighborhood of $C\left(Y_{k}\right) \cup Z_{k}-\left(C\left(Y_{k-1}\right) \cup Z_{k-1}\right)$, there will only be finitely many points in $C\left(Z_{k}\right)-C\left(Z_{k-1}\right)$ for which an object is non-zero. Thus, we could define $f$ to be the identity on $C\left(Y_{k}\right) \cup Z_{k} \cup C\left(Y_{k-1}\right)$ and to send every other point to a chosen point in $\underline{\mathrm{E}} \Gamma^{G} \times(0,1)$. 
Now assume that such sequences exist. Choose a representative $x$, for each orbit not contained in $C\left(Z_{k-1}\right)$ such that $G_{x}=H_{k}$, and choose $y_{x} \in\left(C\left(Y_{k-1}\right) \cup\right.$ $\left.Z_{k-1}\right)^{H_{k}}$ such that $d\left(x, y_{x}\right)=d\left(x,\left(C\left(Y_{k-1}\right) \cup Z_{k-1}\right)^{H_{k}}\right)$. This is possible by the assumption that there exist sequences consisting of points outside of $C\left(Z_{k-1}\right)$ converging to points in $C\left(Y_{k-1}\right) \cup Z_{k-1}$. Choose $z_{x} \in\left(Z_{k-1}-Y_{k-1}\right)^{H_{k}} \times(0,1)$ such that $d\left(z_{x}, y_{x}\right) \leq d\left(x, C\left(Y_{k-1}\right) \cup Z_{k-1}\right)$. This choice is possible by condition 4 of the main theorem. Define $f:\left(C\left(Z_{k}\right), C\left(Y_{k} \cup Z_{k}\right)\right) \rightarrow\left(C\left(Z_{k}\right), C\left(Y_{k} \cup Z_{k}\right)\right)$ by

$$
f(a)=\left\{\begin{aligned}
g z_{x} & \text { if } a=g x \\
a & \text { if } a \in C\left(Y_{k}\right) \cup Z_{k} \cup C\left(Y_{k-1}\right)
\end{aligned}\right.
$$

This is well-defined since $g_{1} x=g_{2} x$ if and only if $g_{2}^{-1} g_{1} \in H_{k}$. Thus, $f$ is $G$ equivariant. As mentioned before, $f$ is not eventually continuous. More precisely, the second and third conditions of eventually continuous maps are not satisfied. The second condition ensures that the induced functor sends objects to objects. To replace this condition, we show that the inverse image under $f$ of a compact set in $C\left(Z_{k-1}\right)-\left(C\left(Y_{k-1}\right) \cup Z_{k-1}\right)$ contains only finitely many points over which an object can be non-zero. We do this as follows.

Let $w \in C\left(Z_{k-1}\right)-\left(C\left(Y_{k-1}\right) \cup Z_{k-1}\right)$ and $d>0$ be given such that $0<d<$ $d\left(w, C\left(Y_{k-1}\right) \cup Z_{k-1}\right)$. Let

$$
U=\left\{z \in C\left(Z_{k}\right) \mid d\left(z, C\left(Y_{k-1}\right) \cup Z_{k-1}\right)<d\left(w, C\left(Y_{k-1}\right) \cup Z_{k-1}-d\right)\right\} .
$$

If $B_{d}(w)$ is the open ball in $C\left(Z_{k-1}\right)$ about $w$ of radius $d$, then $f^{-1}\left(\overline{B_{d}(w)}\right) \subseteq$ $C\left(Z_{k}\right)-U$. To see why, let $a \in f^{-1}\left(\overline{B_{d}(w)}\right)$. If $a \in C\left(Z_{k-1}\right)$ then $a=f(a) \in$ $\overline{B_{d}(w)}$. If $a \notin C\left(Z_{k-1}\right)$ then $a=g x$, where $x$ is the chosen representative, and $d\left(w, C\left(Y_{k-1}\right) \cup Z_{k-1}\right)-d \leq d\left(g z_{x}, C\left(Y_{k-1}\right) \cup Z_{k-1}\right)=d\left(z_{x}, C\left(Y_{k-1}\right) \cup Z_{k-1}\right) \leq$ $d\left(z_{x}, C\left(Y_{k-1}^{H_{k}}\right) \cup Z_{k-1}^{H_{k}}\right) \leq d\left(z_{x}, y_{x}\right) \leq d\left(x, C\left(Y_{k-1}\right) \cup Z_{k-1}\right)=d\left(a, C\left(Y_{k-1}\right) \cup Z_{k-1}\right)$. Given an object $H$, there is a neighborhood $V \subseteq C\left(Z_{k}\right)$ of $C\left(Y_{k}\right) \cup Z_{k}-\left(C\left(Y_{k-1}\right) \cup\right.$ $\left.Z_{k-1}\right)$ on which $H$ is zero. Therefore,

$$
\left\{a \in \overline{f^{-1}\left(\overline{B_{d}(w)}\right)} \mid H_{a} \neq 0\right\} \subset\left(C\left(Z_{k}\right)-U\right) \cap\left(C\left(Z_{k}\right)-V\right) .
$$

Since $\left(C\left(Z_{k}\right)-U\right) \cap\left(C\left(Z_{k}\right)-V\right) \subseteq C\left(Z_{k}\right)-\left(C\left(Y_{k} \cup Z_{k}\right)\right.$ is compact, this set is finite.

The third condition on eventually continuous maps is that they are continuous on the boundary. This condition guarantees that the image under the induced functor of a continuously controlled morphism is continuously controlled. Since objects are zero on a neighborhood of $C\left(Y_{k}\right) \cup Z_{k}-\left(C\left(Y_{k-1}\right) \cup Z_{k-1}\right)$, it suffices 
to show that $f$ is continuous on $C\left(Y_{k-1}\right) \cup Z_{k-1}$. Let $\left\{x_{n}\right\}$ be a sequence in $C\left(Z_{k}\right)-C\left(Z_{k-1}\right)$ converging to $y \in C\left(Y_{k-1}\right) \cup Z_{k-1}$. Since $G$ is a finite group, all but finitely many terms in the sequence must be contained in a subsequence of the form $\left\{g x_{m}\right\}$ for a fixed $g \in G$, where each $x_{k}$ is the chosen representative of its orbit. Since $\left\{x_{m}\right\}$ converges to $g^{-1} y$ and $G_{x_{m}}=H_{k}, g^{-1} y$ is fixed by $H_{k}$. Thus, $\left\{y_{x_{m}}\right\}$ converges to $g^{-1} y$. Therefore, $\left\{z_{x_{m}}\right\}$ converges to $g^{-1} y$ and $\left\{f\left(g x_{m}\right)\right\}=\left\{g z_{x_{m}}\right\}$ converges to $y=f(y)$. Since $\left.f\right|_{C\left(Y_{k}\right) \cup Z_{k}}=\operatorname{id}_{C\left(Y_{k}\right) \cup Z_{k}}$, $f$ induces a functor that is an inverse to the inclusion functor up to natural equivalence as in Lemma 3.1.

This completes the proof of part (i). Part (ii) is proved the same way replacing cones with suspensions.

Lemma 4.6. Let $1 \leq k \leq m+1$. Then:

i. $C_{r}^{*} \mathcal{B}_{\mathbb{C}}^{G}\left(C\left(Z_{k}\right)\right)^{>Z_{k-1}} \cong C_{r}^{*} \mathcal{B}_{\mathbb{C} H_{k}}\left(C\left(Z_{k} / G\right)\right)^{>Z_{k-1} / G}$

ii. $C_{r}^{*} \mathcal{B}_{\mathbb{C}}^{G}\left(\Sigma\left(Z_{k}\right)\right)^{>Z_{k-1}} \cong C_{r}^{*} \mathcal{B}_{\mathbb{C} H_{k}}\left(\Sigma\left(Z_{k} / G\right)\right)^{>Z_{k-1} / G}$

Proof. For every $z \in C\left(Z_{k} / G\right)-C\left(Z_{k-1} / G\right)$ choose $x_{z} \in p^{-1}(z)$ so that $G_{x_{z}}=$ $H_{k}$, where $p: C\left(Z_{k}\right) \rightarrow C\left(Z_{k} / G\right)$ denotes the quotient map. Suppose $H$ and $K$ are objects in $C_{r}^{*} \mathcal{B}_{\mathbb{C}}^{G}\left(C\left(Z_{k}\right)\right)$ that are zero over points in $C\left(Z_{k-1}\right)$ and that $T: H \rightarrow K$ is a continuously controlled morphism such that:

$$
T_{y^{\prime}}^{x^{\prime}}=\left\{\begin{array}{cl}
g T_{y}^{x} g^{-1} & \text { if } x^{\prime}=g x \text { and } y^{\prime}=g y \\
0 & \text { otherwise }
\end{array}\right.
$$

where $G_{x}=G_{y}=H_{k}$. If $T_{G y}^{G x}: H_{G x} \rightarrow K_{G y}$ is non-zero, where $H_{G x}=$ $\bigoplus_{x^{\prime} \in G x} H_{x^{\prime}}$, then the non-zero components of $T_{G y}^{G x}$ are all unitarily equivalent to $T_{y}^{x}$. We shall call $T_{y}^{x}$ a generating component. Note that the equivariance of $T$ implies that $T_{y}^{x}: H_{x} \rightarrow K_{y}$ is $H_{k}$-equivariant. Define the object $\bar{H}$ in $C_{r}^{*} \mathcal{B}_{\mathbb{C} H_{k}}\left(C\left(Z_{k} / G\right)\right)$ as follows: $\bar{H}_{z}=H_{x_{z}}$ if $z \notin C\left(Z_{k-1}\right)$ and is zero otherwise. Define the morphism $\bar{T}: \bar{H} \rightarrow \bar{K}$ as follows: $\bar{T}_{w}^{z}=h^{-1} T_{h x_{w}}^{g x_{z}} g$, where $T_{h x_{w}}^{g x_{z}}$ is a generating component. This assignment is well-defined since $T$ is equivariant. Note that $\|\bar{T}\|=\|T\|$, so $\bar{T}$ will be bounded if and only if $T$ is bounded. This also implies that if $S=\lim _{n} T_{n}$ is a limit of such morphisms, then $\bar{S}=\lim _{n} \bar{T}_{n}$ is well-defined.

The scenario described above tells the entire story. The reason is that since we are taking germs away from $C\left(Y_{k-1}\right) \cup Z_{k-1}$ (resp. $C\left(Y_{k-1} / G\right) \cup Z_{k-1} / G$ ), the 
components of continuously controlled morphisms need to become small. This implies that a morphism in $\mathcal{B}_{\mathbb{C}}^{G}\left(C\left(Z_{k}\right)\right)^{>Z_{k-1}}\left(\operatorname{resp} . \mathcal{B}_{\mathbb{C} H_{k}}\left(C\left(Z_{k} / G\right)\right)^{>Z_{k-1} / G}\right.$ ) will have a representative that is zero on $C\left(Z_{k-1}\right)$ (resp. $C\left(Z_{k-1} / G\right)$ ). Therefore, it is irrelevant what the objects over $C\left(Z_{k-1}\right)$ and $C\left(Z_{k-1} / G\right)$ are. Since we are working with a finite group action, non-zero components of a continuously controlled morphism have the same isotropy, namely a conjugate of $H_{k}$. This also tells us that the equivariance of morphisms in $C_{r}^{*} \mathcal{B}_{\mathbb{C}}^{G}\left(C\left(Z_{k}\right)\right)^{>Z_{k-1}}$ implies that there is only one choice when lifting a continuously controlled morphism from $C_{r}^{*} \mathcal{B}_{\mathbb{C} H_{k}}\left(C\left(Z_{k} / G\right)\right)^{>Z_{k-1} / G}$. Furthermore, if two morphisms $S$ and $S^{\prime}$, are limits of continuously controlled morphisms of the type described above and are identified in $C_{r}^{*} \mathcal{B}_{\mathbb{C}}^{G}\left(C\left(Z_{k}\right)\right)^{>Z_{k-1}}$, then $\bar{S}$ will be related to $\bar{S}^{\prime}$. Since every morphism can be identified with a morphism that is such a limit, we have defined a functor from $C_{r}^{*} \mathcal{B}_{\mathbb{C}}^{G}\left(C\left(Z_{k}\right)\right)^{>Z_{k-1}}$ to $C_{r}^{*} \mathcal{B}_{\mathbb{C} H_{k}}\left(C\left(Z_{k} / G\right)\right)^{>Z_{k-1} / G}$ that is an equivalence of categories. The same argument proves the second part of this lemma, replacing cones with suspensions.

Lemma 4.7. For each $k, 1 \leq k \leq m+1$,

$$
\mathbb{K}^{\mathrm{Top}}\left(C_{r}^{*} \mathcal{B}_{\mathbb{C} H_{k}}\left(C\left(Z_{k} / G\right)\right)^{>Z_{k-1} / G}\right) \simeq \mathbb{K}^{\mathrm{Top}}\left(C_{r}^{*} \mathcal{B}_{\mathbb{C} H_{k}}\left(\Sigma\left(Z_{k} / G\right)\right)^{>Z_{k-1} / G}\right) .
$$

Proof. The proof proceeds just as in [Ros04, Lemma 7.5]. An unequivariant version of the proof of Lemma 4.5 proves that

$$
C_{r}^{*} \mathcal{B}_{\mathbb{C} H_{k}}\left(C\left(Z_{k} / G\right)\right)_{Z_{k-1} / G} \cong C_{r}^{*} \mathcal{B}_{\mathbb{C} H_{k}}\left(C\left(Z_{k-1} / G\right)\right)
$$

and

$$
C_{r}^{*} \mathcal{B}_{\mathbb{C} H_{k}}\left(\Sigma\left(Z_{k} / G\right)\right)_{Z_{k-1} / G} \cong C_{r}^{*} \mathcal{B}_{\mathbb{C} H_{k}}\left(\Sigma\left(Z_{k-1} / G\right)\right) .
$$

Therefore, we have the following commutative diagram:

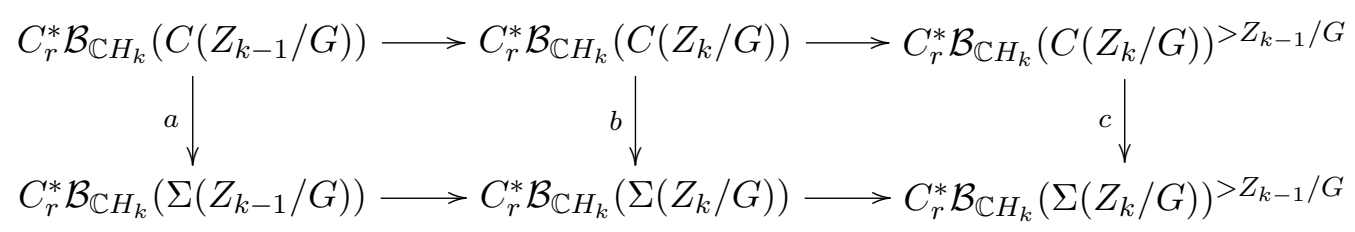

Since $Z_{k-1}$ and $Z_{k}$ are contractible, Corollary 3.12 and Proposition $3.10 \mathrm{imply}$ that both $a$ and $b$ induce weak homotopy equivalences. Each row in the diagram is a Karoubi filtration. Thus, the Five Lemma shows that $c$ also induces a weak homotopy equivalence.

We are now ready to prove Theorem 4.4. 
Proof of Theorem 4.4. Since $G$ acts trivially on $X^{G}$,

$$
C_{r}^{*} \mathcal{B}_{\mathbb{C}}^{G}\left(C\left(X^{G}\right)\right) \cong C_{r}^{*} \mathcal{B}_{\mathbb{C} G}\left(C\left(X^{G}\right)\right)
$$

and

$$
C_{r}^{*} \mathcal{B}_{\mathbb{C}}^{G}\left(\Sigma\left(X^{G}\right)\right) \cong C_{r}^{*} \mathcal{B}_{\mathbb{C} G}\left(\Sigma\left(X^{G}\right)\right)
$$

Since $X^{G}$ is contractible, Proposition 3.10 implies

$$
\mathbb{K}^{\mathrm{Top}}\left(C_{r}^{*} \mathcal{B}_{\mathbb{C} G}\left(C\left(X^{G}\right)\right)\right) \simeq \mathbb{K}^{\mathrm{Top}}\left(C_{r}^{*} \mathcal{B}_{\mathbb{C} G}\left(\Sigma\left(X^{G}\right)\right)\right) .
$$

Therefore, $\mathbb{K}^{\operatorname{Top}}\left(C_{r}^{*} \mathcal{B}_{\mathbb{C}}^{G}\left(C\left(X^{G}\right)\right)\right) \simeq \mathbb{K}^{\operatorname{Top}}\left(C_{r}^{*} \mathcal{B}_{\mathbb{C}}^{G}\left(\Sigma\left(X^{G}\right)\right)\right)$. This completes the base case of the induction.

Assume now that $\mathbb{K}^{\mathrm{Top}}\left(C_{r}^{*} \mathcal{B}_{\mathbb{C}}^{G}\left(C\left(Z_{k-1}\right)\right)\right) \simeq \mathbb{K}^{\mathrm{Top}}\left(C_{r}^{*} \mathcal{B}_{\mathbb{C}}^{G}\left(\Sigma\left(Z_{k-1}\right)\right)\right)$. We want to show that $\mathbb{K}^{\mathrm{Top}}\left(C_{r}^{*} \mathcal{B}_{\mathbb{C}}^{G}\left(C\left(Z_{k}\right)\right)\right) \simeq \mathbb{K}^{\mathrm{Top}}\left(C_{r}^{*} \mathcal{B}_{\mathbb{C}}^{G}\left(\Sigma\left(Z_{k}\right)\right)\right)$. Consider the following commutative diagram:

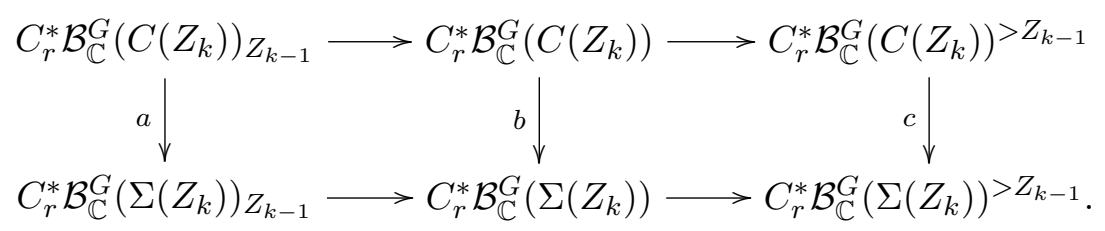

By Lemma 4.5, Proposition 3.10, and the induction hypothesis, $a$ induces a weak homotopy equivalence. By Lemmas 4.6 and $4.7, c$ induces a weak homotopy equivalence. Since each row in the diagram is a Karoubi filtration, the Five Lemma shows that $b$ also induces a weak homotopy equivalence.

This completes the proof of the main theorem.

\section{REFERENCES}

[Ad94] S. Adams, Boundary amenability for word hyperbolic groups and an application to smooth dynamics of simple groups, Topology 33 (1994), 765-783.

[BR07] A. Bartels and D. Rosenthal, On the algebraic $K$ - and L-theory of groups with finite asymptotic dimension, J. Reine Agnew. Math. 612 (2007), 35-57.

[BCH94] P. Baum, A. Connes, and N. Higson, Classifying space for proper actions an K-theory of group $C^{*}$-algebras, $C^{*}$-algebras: 1943-1993 (San Antonio, TX, 1993), Contemp. Math., vol, 167, Amer. Math. Soc., Providence, RI, 1994, 240-291.

[Bre72] G. Bredon, Introduction to Compact Transformation Groups, Acad. Press 1972.

[CP95] G. Carlsson and E. K. Pedersen, Controlled algebra and the Novikov conjectures for $K$ - and L- theory, Topology 34 (1995), 731-758. 
[Con60] P. E. Conner, Retraction properties of the orbit space of a compact topological transformation group, Duke Math. J. 27 (1960), 341-357.

[DL98] J. F. Davis and W. Lück, Spaces over a category and assembly maps in isomorphism conjectures in $K$ - and $L$ - theory, K-theory 15 (1998), 201-252.

[EH76] D. A. Edwards and H. M. Hastings, Čech and Steenrod Homotopy Theories with Applications to Geometric Topology, Lecture Notes in Mathematics, vol. 542, SpringerVerlag, Berlin-New York, 1976.

[FH81] F. T. Farrell and W. C. Hsiang, On Novikov's conjecture for non-positively curved manifolds, I, Ann. of Math. 113(2) (1981), 197-209.

[FJ93] F. T. Farrell and L. E. Jones, Isomorphism conjectures in algebraic $K$-theory, J. Amer. Math. Soc. 6 (1993), no. 3, 249-297.

[Flo51] E. E. Floyd, Some retraction properties of the orbit decomposition space of periodic maps, Amer. J. Math. 73 (1951), 363-367.

[HP04] I. Hambleton and E. K. Pedersen, Identifying assembly maps in $K$ - and L-theory, Math. Ann. 328, (2004), 27-57.

[Hig00] N. Higson, Bivariant K-theory and the Novikov conjecture, Geom. Funct. Anal. 10 (2000), no. 3, 563-581.

[HPR97] N. Higson, E. K. Pedersen, and J. Roe, $C^{*}$-algebras and controlled topology, $K$-theory 11 (1997), 209-239.

[KKS77] D. S. Kahn, J. Kaminker, and C. Schochet, Generalized homology theories on compact metric spaces, Michigan Math. J. 24 (1977), 203-224.

[Kar70] M. Karoubi, Foncteur derivees et K-theorie, Lecture Notes in Mathematics, vol. 136, Springer-Verlag, Berlin-New York, 1970.

[Kas88] G. G. Kasparov, Equivariant KK-theory and the Novikov conjecture, Invent. Math. 91 (1988), 147-201.

[LRV03] W. Lück, H. Reich and M. Varisco, Commuting homotopy limits and smash products, $K$-theory 30 (2003), 137-165.

[Mil63] J. Milnor, On the Steenrod homology theory, Novikov Conjectures, Rigidity and Index Theorems Vol. 1 (Oberwolfach, 1993), London Math. Soc. Lecture Notes, vol. 226, Cambridge University Press, Cambridge-New York, 1995, 79-96.

[MY02] I. Mineyev and G. Yu, The Baum-Connes conjecture for hyperbolic groups, Invent. Math. 149 (2002), 97-122.

[Oli76] R. Oliver, A proof of the Conner conjecture, Ann. Math. 103 (1976), 637-644.

[PW85] E. K. Pedersen and C. Weibel, A non-connective delooping of algebraic K-theory, Algebraic and Geometric Topology (Rutgers, 1983), Lecture Notes in Mathematics, vol. 1126, Springer, Berlin, 1985, 166-181.

[Ros04] D. Rosenthal, Splitting with continuous control in algebraic $K$-theory, $K$-theory 32 (2004), 139-166.

[Ros06] D. Rosenthal, Continuous control and the algebraic L-theory assembly map, Forum Math. 18 (2006), 193-209.

[RS05] D. Rosenthal and D. Schütz, On the algebraic $K$ - and L-theory of word hyperbolic groups, Math. Annalen. 332 (2005), 523-532. 
David Rosenthal

Department of Mathematics and Computer Science

St. John's University

Jamaica, NY 11439

USA

E-mail: rosenthd@stjohns.edu 